\title{
TAELMAN $L$-VALUES FOR DRINFELD MODULES OVER TATE ALGEBRAS
}

\author{
OĞUZ GEZMiş
}

\begin{abstract}
In the present paper, we investigate Taelman $L$-values corresponding to Drinfeld modules over Tate algebras of arbitrary rank. Using our results, we also introduce an $L$-series converging in Tate algebras which can be seen as a generalization of Pellarin $L$-series.
\end{abstract}

\section{INTRODUCTION}

Let $p$ be a prime number, and set $q:=p^{l}$ where $l$ is a positive integer. We denote the finite field with $q$ elements by $\mathbb{F}_{q}$. Let $\theta$ be an indeterminate over $\mathbb{F}_{q}$. We set $A:=\mathbb{F}_{q}[\theta]$ and $K:=\mathbb{F}_{q}(\theta)$. We define $A_{+}$to be the set of monic polynomials in $A$, and $A_{+, k}$ to be the subset of $A_{+}$comprising polynomials of degree $k$. We define $\operatorname{ord}_{\infty}$ to be the valuation on $K$ corresponding to the point at $\infty$ defined so that $\operatorname{ord}_{\infty}(\theta)=-1$. We denote the completion of $K$ with respect to $\operatorname{ord}_{\infty}$ by $\mathbb{K}_{\infty}$, and the completion of the algebraic closure of $\mathbb{K}_{\infty}$ by $\mathbb{C}_{\infty}$. We let $|\cdot|_{\infty}$ be the normalized $\infty$-adic norm on $\mathbb{C}_{\infty}$ such that $|\theta|_{\infty}=q$. For any $\mathbb{F}_{q}$-algebra $R$ with an $\mathbb{F}_{q}$-algebra homomorphism $\tau: R \rightarrow R$, we define the noncommutative skew polynomial ring $R\{\tau\}$ and the formal power series ring $R\{\{\tau\}\}$ by the relation $\tau \cdot x=\tau(x) \cdot \tau$ for all $x \in R$. Finally, for any field $k$ and any finite $k[\theta]$-module $M$, we denote the monic generator of the Fitting ideal of $M$ by $[M]_{k[\theta]}$ (see $\S 2.2$ for the details about Fitting ideals).

A Drinfeld $A$-module $\phi$ of rank $r$ (over $A$ ) is an $\mathbb{F}_{q}$-algebra homomorphism

$$
\phi: A \rightarrow A\{\tau\}
$$

defined by

$$
\phi_{\theta}=\theta+\phi_{\theta, 1} \tau+\cdots+\phi_{\theta, r} \tau^{r}
$$

such that $\phi_{\theta, r} \neq 0$. For any integer $s$, the dual Goss $L$-series $L\left(\phi^{\vee}, s-1\right)$ corresponding to $\phi$ is defined by

$$
L\left(\phi^{\vee}, s-1\right)=\sum_{a \in A_{+}} \frac{\mu(a)}{a^{s}},
$$

where $\mu: A_{+} \rightarrow A$ is a multiplicative function depending on $\phi$ (eg. see [8, Lem.3.5]). As an example, if $\phi$ is the Carlitz module $C$ defined by $C_{\theta}=\theta+\tau$, then $\mu(a)=1$ for all $a \in A_{+}$. In [22], Taelman defined a new type of $L$-value $L(\phi, A)$ by

$$
L(\phi, A)=\prod_{f} \frac{[A / f A]_{A}}{[\phi(A / f A)]_{A}},
$$

Date: July 17, 2018.

2010 Mathematics Subject Classification. Primary 11M38; Secondary 11G09, 11R58.

$K e y$ words and phrases. Drinfeld modules, Tate algebras, Pellarin $L$-series, Taelman $L$-values.

This project was partially supported by NSF Grant DMS-1501362. 
where the product runs over irreducible polynomials $f \in A_{+}$(see $\S 2.2$ and 2.3 for the details). Moreover, Taelman observed [22, Rem. 5] that $L(\phi, A)$ is also related to the dual Goss $L$ series by

$$
L\left(\phi^{\vee}, 0\right)=\sum_{a \in A_{+}} \frac{\mu(a)}{a}=L(\phi, A) .
$$

We define the Tate algebra $\mathbb{T}_{n}$ as the set of all elements of the form $\sum x_{i_{1}, \ldots, i_{n}} z_{1}^{i_{1}} \ldots z_{n}^{i_{n}} \in$ $\mathbb{C}_{\infty}\left[\left[z_{1}, \ldots, z_{n}\right]\right]$ such that $\left|x_{i_{1}, \ldots, i_{n}}\right|_{\infty} \rightarrow 0$ as $i_{1}+\cdots+i_{n} \rightarrow \infty$. For $g=\sum x_{i_{1}, \ldots, i_{n}} z_{1}^{i_{1}} \ldots z_{n}^{i_{n}} \in$ $\mathbb{T}_{n}$, the Gauss norm $\|\cdot\|$ on $\mathbb{T}_{n}$ is defined by $\|g\|:=\sup \left\{\left|x_{i_{1}, \ldots, i_{n}}\right|_{\infty} \mid i_{j} \in \mathbb{N}\right\}$. We denote its associated valuation by ord such that $\operatorname{ord}(g):=\min \left\{\operatorname{ord}_{\infty}\left(x_{i_{1}, \ldots, i_{n}}\right) \mid i_{j} \in \mathbb{N}\right\}$. For any $f=g / h \in \mathbb{C}_{\infty}\left(z_{1}, \ldots, z_{n}\right)$ such that $g, h \in \mathbb{C}_{\infty}\left[z_{1}, \ldots, z_{n}\right]$ with $h \neq 0$, we set ord $(f):=$ $\operatorname{ord}(g)-\operatorname{ord}(h)$ and denote the completion of $\mathbb{C}_{\infty}\left(z_{1}, \ldots, z_{n}\right)$ with respect to the valuation ord by $\widetilde{\mathbb{T}_{n}}$. Now set $\mathbb{A}:=\mathbb{F}_{q}\left(z_{1}, \ldots, z_{n}\right)[\theta]$. For $1 \leq k \leq n$, define $\ell_{0}\left(z_{k}\right):=1$, and for $i \geq 1$, $\ell_{i}\left(z_{k}\right):=\prod_{j=0}^{i-1}\left(z_{k}-\theta^{q^{j}}\right)$. Following the work of Anglès and Tavares Ribeiro in [6, §3], we define a Drinfeld $\mathbb{A}$-module $\varphi$ of rank $r$ as an $\mathbb{F}_{q}\left(z_{1}, \ldots, z_{n}\right)$-algebra homomorphism

$$
\varphi: \mathbb{A} \rightarrow \mathbb{A}\{\tau\}
$$

given by

$$
\varphi_{\theta}=\sum_{i=0}^{r} \varphi_{\theta, i} \tau^{i}=\sum_{i=0}^{r} \ell_{i}\left(z_{1}\right) \ldots \ell_{i}\left(z_{n}\right) \phi_{\theta, i} \tau^{i} .
$$

The infinite series $\sum_{i \geq 0} \beta_{i} \tau^{i} \in \mathbb{T}_{n}[[\tau]]$ induces the exponential function of $\varphi$

$$
\exp _{\varphi}: \widetilde{\mathbb{T}_{n}} \rightarrow \widetilde{\mathbb{T}_{n}}
$$

defined by $\exp _{\varphi}(f)=\sum_{i \geq 0} \beta_{i} \tau^{i}(f)$ for all $f \in \widetilde{\mathbb{T}_{n}}$. Furthermore, the series $\sum_{i \geq 0} \xi_{i} \tau^{i} \in \mathbb{T}_{n}[[\tau]]$ induces the logarithm function of $\varphi$

$$
\log _{\varphi}: \widetilde{\mathbb{T}_{n}} \rightarrow \widetilde{\mathbb{T}_{n}}
$$

defined by $\log _{\varphi}(f)=\sum_{i \geq 0} \xi_{i} \tau^{i}(f)$, and it satisfies $\exp _{\varphi}\left(\log _{\varphi}(f)\right)=\log _{\varphi}\left(\exp _{\varphi}(f)\right)=f$ for any $f \in \widetilde{\mathbb{T}_{n}}$ within the radius of convergence of $\log _{\varphi}$ (see $\S 3.1$ for more details about coefficients $\beta_{i}$ and $\xi_{i}$.

After Taelman $L$-values were introduced for Drinfeld $A$-modules, Anglès, Pellarin, and Tavares Ribeiro developed the theory in [3, §5] for the deformation of the Carlitz module $C$. Later on, Anglès and Tavares Ribeiro extended the theory for a Drinfeld $\mathbb{A}$-module $\varphi$ of arbitrary rank in [6], and defined the Taelman $L$-value corresponding to $\varphi$ by the infinite product

$$
L(\varphi, \mathbb{A})=\prod_{f} \frac{[\mathbb{A} / f \mathbb{A}]_{\mathbb{A}}}{[\varphi(\mathbb{A} / f \mathbb{A})]_{\mathbb{A}}}
$$

where $f$ is irreducible in $A_{+}$(see $\S 4.1$ for explicit definitions). Furthermore, Anglès, Pellarin, and Tavares Ribeiro [3, Prop. 5.9] proved that if $\tilde{C}$ is a Drinfeld $\mathbb{A}$-module defined by $\tilde{C}_{\theta}=\theta+\left(z_{1}-\theta\right) \ldots\left(z_{n}-\theta\right) \tau$, then

$$
L(\tilde{C}, \mathbb{A})=\sum_{a \in A_{+}} \frac{a\left(z_{1}\right) \ldots a\left(z_{n}\right)}{a} .
$$


In the present paper, we generalize the work of Anglès, Pellarin, and Tavares Ribeiro in [3] to special Drinfeld $\mathbb{A}$-modules of arbitrary rank which are defined by Anglès and Tavares Ribeiro in [6, §3]. Our first result is the following theorem (stated as Theorem 3.3 later).

Theorem 1.1. Let $\phi$ be a Drinfeld A-module defined as in (1) and $\varphi$ be a Drinfeld $\mathbb{A}$-module defined as in (2). Let $L(\varphi, \mathbb{A})$ be the Taelman L-value corresponding to $\varphi$. Then

$$
L(\varphi, \mathbb{A})=\sum_{a \in A_{+}} \frac{\mu(a) a\left(z_{1}\right) \ldots a\left(z_{n}\right)}{a} .
$$

In order to prove Theorem 1.1 , we need to analyze $[\varphi(\mathbb{A} / f \mathbb{A})]_{\mathbb{A}}$ for all monic irreducible polynomials $f \in A$. For a Drinfeld $A$-module of arbitrary rank, this was done by using Tate modules (see [8], [14]). The main difficulty in our case is that we do not yet understand Tate modules for Drinfeld $\mathbb{A}$-modules. Therefore, to prove the theorem, we follow a different direction, and consider Drinfeld $\mathbb{F}_{q}(z)[\theta]$-modules ( $z$ an indeterminate over $\mathbb{F}_{q}$ ) which were first introduced by Anglès and Tavares Ribeiro in [6, §2.2] (see §2.4).

Let $\omega_{n} \in \mathbb{T}_{n}^{\times}$be an Anderson-Thakur type element (see $\S 2.1$ for the definition). Anglès and Tavares Ribeiro [6, §3.2] proved that $\exp _{\varphi}(L(\varphi, \mathbb{A})) \in A\left[z_{1}, \ldots, z_{n}\right]$. Combining this result with Theorem 1.1 and by a small calculation (see Remark 4.4), we deduce the following corollary.

Corollary 1.2. Let us set

$$
P_{\phi}\left(z_{1}, \ldots, z_{n}\right):=\exp _{\varphi}\left(\sum_{a \in A_{+}} \frac{\mu(a) a\left(z_{1}\right) \ldots a\left(z_{n}\right)}{a}\right) .
$$

Then $P_{\phi}\left(z_{1}, \ldots, z_{n}\right) \in A\left[z_{1}, \ldots, z_{n}\right]$. If $P_{\phi}\left(z_{1}, \ldots, z_{n}\right)$ is within the radius of convergence of $\log _{\varphi}$, then

$$
\sum_{a \in A_{+}} \frac{\mu(a) a\left(z_{1}\right) \ldots a\left(z_{n}\right)}{a}=\log _{\varphi}\left(P\left(z_{1}, \ldots, z_{n}\right)\right)=\frac{\log _{\phi}\left(\omega_{n} P_{\phi}\left(z_{1}, \ldots, z_{n}\right)\right)}{\omega_{n}} .
$$

As an immediate consequence of [3, Rem. 5.13], Anglès, Pellarin, and Tavares Ribeiro proved that if $0 \leq n \leq q-1$, then

$$
L(\tilde{C}, \mathbb{A})=\sum_{a \in A_{+}} \frac{a\left(z_{1}\right) \ldots a\left(z_{n}\right)}{a}=\frac{\log _{C}\left(\omega_{n}\right)}{\omega_{n}} .
$$

In the following result (stated as Corollary 4.3 later), we generalize the identity in (3) by analyzing when the polynomial $P_{\phi}\left(z_{1}, \ldots, z_{n}\right)$ in Corollary 1.2 is equal to 1 , and for which Drinfeld $\mathbb{A}$-modules $\varphi$ as in (2), 1 is within the radius of convergence of $\log _{\varphi}$. Before stating the following result, we set $\beta:=\max \left\{\operatorname{deg}_{\theta}\left(\phi_{\theta, i}\right) \mid 1 \leq i \leq r\right\}$.

Corollary 1.3. Let $\phi$ be a Drinfeld $A$-module defined as in (1), and $\varphi$ be a Drinfeld $\mathbb{A}$-module defined as in (2). If $0 \leq n \leq q / r-(1+2 \beta)$, then

$$
L(\varphi, \mathbb{A})=\sum_{a \in A_{+}} \frac{\mu(a) a\left(z_{1}\right) \ldots a\left(z_{n}\right)}{a}=\frac{\log _{\phi}\left(\omega_{n}\right)}{\omega_{n}} .
$$

The importance of Corollary 1.3 is that it links $\omega_{n}$, which can be defined in terms of the exponential function of the Carlitz module $C$ (see Example 4.6), and the logarithm function 
of a Drinfeld $A$-module $\phi$ of an arbitrary rank. In other words, the Taelman $L$-value reveals a non-trivial link between Drinfeld $A$-modules over $A$ of arbitrary rank and the Carlitz module.

The reader will no doubt observe that the special value in Theorem 1.1 is also the value of a Pellarin $L$-series. In [20], Pellarin introduced the following $L$-series

$$
L\left(z_{1}, \ldots, z_{n}, s\right)=\sum_{d \geq 0} \sum_{a \in A_{+, d}} a\left(z_{1}\right) \ldots a\left(z_{n}\right) a^{-s}
$$

for any integer $s>0$ which is called Pellarin L-series. Now, for any $a \in A_{+}$, set $\langle a\rangle:=$ $a \theta^{-\operatorname{deg}_{\theta}(a)}$. In [17], Goss defined another type of Pellarin $L$-series

$$
L\left(z_{1}, \ldots, z_{n} ; x, y\right)=\sum_{d \geq 0} x^{-d} \sum_{a \in A_{+, d}} a\left(z_{1}\right) \ldots a\left(z_{n}\right)\langle a\rangle^{y}
$$

for any $(x, y) \in \mathbb{C}_{\infty}^{\times} \times \mathbb{Z}_{p}:=\mathbb{S}_{\infty}$ where we call $\mathbb{S}_{\infty}$ Goss' upper half plane (see $\S 4$ for details). One can observe that $L\left(z_{1} \ldots, z_{n}, s\right)=L\left(z_{1}, \ldots, z_{n} ; \theta^{s},-s\right)$. Moreover, when $n=1$, Goss proved [17, Thm. 1] that $L\left(z_{1}, \ldots, z_{n} ; x, y\right)$ is entire on $\mathbb{C}_{\infty} \times \mathbb{S}_{\infty}$, and Anglès and Pellarin [2, Prop. 6] proved the same result for arbitrary $n$.

Let $\phi$ be a Drinfeld $A$-module as in (11). We define

$$
L\left(\phi^{\vee}, z_{1}, \ldots, z_{n} ; x, y\right)=\sum_{d \geq 0} x^{-d} \sum_{a \in A_{+, d}} \mu(a) a\left(z_{1}\right) \ldots a\left(z_{n}\right)\langle a\rangle^{y}
$$

for any $(x, y) \in \mathbb{S}_{\infty}$. We further set

$$
L\left(\phi^{\vee}, z_{1} \ldots, z_{n}, s\right):=L\left(\phi^{\vee}, z_{1}, \ldots, z_{n} ; \theta^{s},-s\right)=\sum_{a \in A_{+}} \frac{\mu(a) a\left(z_{1}\right) \ldots a\left(z_{n}\right)}{a^{s}} .
$$

for any integer $s>0$. Note that $L\left(C^{\vee}, z_{1} \ldots, z_{n}, s\right)=L\left(z_{1}, \ldots, z_{n}, s\right)$, and by Theorem 3.3 , we have $L\left(\phi^{\vee}, z_{1} \ldots, z_{n}, 1\right)=L(\varphi, \mathbb{A})$.

As a generalization of the results mentioned above for Pellarin $L$-series in (5), we prove the following theorem (stated as Theorem 5.1 later).

Theorem 1.4. The infinite series $L\left(\phi^{\vee}, z_{1}, \ldots, z_{n} ; x, y\right)$ can be analytically continued to an entire function on $\mathbb{C}_{\infty}^{n} \times \mathbb{S}_{\infty}$.

One of the reasons to prove Theorem 5.1 is that the process of the proof provides us some remarkable results about Drinfeld $A$-modules over $A$ of arbitrary rank. Our method of proving Theorem 5.1 relies on a multivariable version of the log-algebraicity theorem for Drinfeld $A$-modules over $A$ of arbitrary rank which can be stated as follows (later stated as Theorem [5.3).

Theorem 1.5. Let $\phi$ be a Drinfeld $A$-module as in (11), and let $X_{1}, \ldots, X_{n}, w$ be indeterminates. The infinite series

$$
\exp _{\phi}\left(\sum_{a \in A_{+}} \frac{\mu(a) C_{a}\left(X_{1}\right) \ldots C_{a}\left(X_{n}\right)}{a} w^{q^{\operatorname{deg}_{\theta}(a)}}\right) \in K\left[X_{1}, \ldots, X_{n}\right][[w]]
$$

is actually in $A\left[X_{1}, \ldots, X_{n}, w\right]$.

The proof of Theorem 1.5 depends on the method of Anglès, Pellarin, and Tavares Ribeiro given in [3, §8] to prove the multivariable log-algebraicity theorem for the Carlitz module $C$. We also remark that the one variable version of Theorem 1.5 was proved by Chang, El-Guindy, and Papanikolas in [8] using the method of Anderson in [1]. 
One of the consequences of Theorem 1.5 is that we provide vanishing results on power sums twisted by the function $\mu: A_{+} \rightarrow A$, such as the following one (stated as Lemma 5.5 later) used to prove Theorem 1.4.

Lemma 1.6. For $k>r(n+\beta) /(q-1)$, the series

$$
H_{k, n-1}:=\sum_{a \in A_{+, k}} \mu(a) a\left(z_{1}\right) \ldots a\left(z_{n-1}\right)
$$

vanishes.

The outline of the paper can be stated as follows. In $\S 2$, we give further definitions which will be used throughout the paper, and review Taelman $L$-values corresponding to Drinfeld $A$-modules. In $\S 3$, we calculate Taelman $L$-values corresponding to special Drinfeld modules over Tate algebras introduced by Anglès and Tavares Ribeiro in [6, §3]. In $\S 4$, we define the $L$-series in (6) corresponding to Drinfeld $A$-module $\phi$, and relate its values to Taelman $L$ values. Finally, $\S 5$ occupies the proof of Theorem 1.4.

\section{ACKNOWLEDGEMENT}

The author is thankful to Matthew A. Papanikolas for useful suggestions and fruitful discussions.

\section{Notations And Preliminaries}

We define the Tate algebra $\mathbb{T}_{n, t}$ by considering the extra indeterminate $t$, and denote the completion of $\mathbb{C}_{\infty}\left(z_{1}, \ldots, z_{n}, t\right)$ with respect to the valuation ord by $\widetilde{\mathbb{T}_{n, t}}$.

We define $\tau: \widetilde{\mathbb{T}_{n, t}} \rightarrow \widetilde{\mathbb{T}_{n, t}}$ as a homomorphism of $\mathbb{F}_{q}\left(z_{1}, \ldots, z_{n}, t\right)$-algebras such that $\tau(\theta)=$ $\theta^{q}$, and its restriction on $\widetilde{\mathbb{T}_{n}}$ can be defined similarly. Finally, for any $f \in A$ and any $\alpha \in \mathbb{C}_{\infty}$, we denote the evaluation of $f$ at $\theta=\alpha$ by $f(\alpha)$, and when $X$ is an indeterminate, we use $f(X)$ for the evaluation of $f$ at $\theta=X$.

2.1. Anderson-Thakur type elements. Let $\alpha=\left(z_{1}-\theta\right)\left(z_{2}-\theta\right) \ldots\left(z_{n}-\theta\right) \in \mathbb{T}_{n}$. Choose $y=(-\theta)^{n}$. Then we observe that $\|y-\alpha\|<\|\alpha\|$, and that

$$
\left\|\frac{y^{q^{j}}}{\tau^{j}(\alpha)}-1\right\|=\left\|\frac{\tau^{j}(y-\alpha)}{\tau^{j}(\alpha)}\right\| \rightarrow 0 \quad \text { as } \quad j \rightarrow \infty .
$$

Now fix a $(q-1)^{\text {st }}$ root of $y$, and let $\xi=y^{\frac{1}{q-1}}$. Then we define an Anderson-Thakur type element $\omega_{n}$ by the infinite product

$$
\omega_{n}:=\xi \prod_{j=0}^{\infty} \frac{y^{q^{j}}}{\tau^{j}(\alpha)}
$$

We refer the reader to [3] and [15] for further readings about Anderson-Thakur type elements. 
2.2. Fitting Ideals. Let $k$ be any field, and set $R:=k[\theta]$. Let $M$ be a finite $R$-module given as the direct sum $\bigoplus_{i=1}^{n} R / f_{i} R$ of the quotient modules where $f_{i}$ is monic in $R$ for $1 \leq i \leq n$. Then we set a monic polynomial $[M]_{R}:=f_{1} \dot{f}_{2} \ldots f_{n}$, and we call the principal ideal generated by $[M]_{R}$ in $R$ the Fitting ideal of $M$. As an example, we observe that $[R / f R]_{R}=f$ for any monic polynomial $f \in R$. We also note that by $[6, \S 2.1],[M]_{R}$ can be also defined by

$$
[M]_{R}=\operatorname{det}_{k[X]}\left((1 \otimes X) I d-(\theta \otimes 1) \mid M \otimes_{k} k[X]\right)_{\mid X=\theta},
$$

which can be seen as the evaluation at $X=\theta$ of the characteristic polynomial of the action of $\theta$ on the $R$-module $M \otimes_{k} k$ given by $\theta \cdot(x \otimes 1)=\theta \cdot x \otimes 1$ for all $x \in M$. We refer the reader to [6] and [22] for details about Fitting ideals.

2.3. Drinfeld $A$-Modules. Let $\phi$ be a Drinfeld $A$-module defined as in (11). The exponential series

$$
\exp _{\phi}=\sum_{j \geq 0} \alpha_{j} \tau^{j} \in \mathbb{C}_{\infty}[[\tau]]
$$

is defined so that $\alpha_{0}=1$ and $\exp _{\phi} a=\phi_{a} \exp _{\phi}$ for all $a \in A$. The series induces an $\mathbb{F}_{q}$-linear endomorphism and also an entire function $\exp _{\phi}: \mathbb{C}_{\infty} \rightarrow \mathbb{C}_{\infty}$ defined by $\exp _{\phi}(x)=\sum_{j \geq 0} \alpha_{j} x^{q^{j}}$ for all $x \in \mathbb{C}_{\infty}$.

On the other hand, the logarithm series corresponding to $\phi$ is defined by

$$
\log _{\phi}=\sum_{j \geq 0} \gamma_{j} \tau^{j} \in \mathbb{C}_{\infty}[[\tau]]
$$

such that $\gamma_{0}=1$ and $\log _{\phi} \phi_{a}=a \log _{\phi}$ for all $a \in A$. It induces the logarithm function $\log _{\phi}: \mathbb{C}_{\infty} \rightarrow \mathbb{C}_{\infty}$ which is defined by $\log _{\phi}(x)=\sum_{j \geq 0} \gamma_{j} x^{q^{j}}$ for all $x \in \mathbb{C}_{\infty}$ within the radius of convergence of $\log _{\phi}$. Furthermore, for any $x \in \mathbb{C}_{\infty}$ where $\log _{\phi}(x)$ is defined, we have $\exp _{\phi}\left(\log _{\phi}(x)\right)=\log _{\phi}\left(\exp _{\phi}(x)\right)=x$.

Example 2.1. The Carlitz module $C$ defined by $C_{\theta}=\theta+\tau$ is an example of a rank 1 Drinfeld $A$-module. It has the exponential function $\exp _{C}$ so that $\operatorname{Ker}\left(\exp _{C}\right)=\tilde{\pi} A$ where $\tilde{\pi} \in \mathbb{C}_{\infty}^{\times}$

Let $f \in A_{+}$be an irreducible polynomial. For any element $a \in A$, define $\bar{a}$ such that $a \equiv \bar{a}$ $(\bmod f)$. We define $\bar{\phi}: A \rightarrow A / f A[\tau]$ by

$$
\bar{\phi}_{\theta}=\sum_{i=0}^{r_{0}} \overline{\phi_{\theta, i}} \tau^{i}
$$

such that $\overline{\phi_{\theta, r_{0}}} \neq 0$. Observe that $r_{0}$ depends on the Drinfeld $A$-module $\phi$ and the polynomial $f$. Note also that $0 \leq r_{0} \leq r$. We also set $\phi(A / f A)$ to $A / f A$ whose $A$-module action given by $\theta \cdot f=\bar{\phi}_{\theta}(f)$ for all $f \in A / f A$.

The Taelman $L$-value $L(\phi, A)$ corresponding to $\phi$ is defined by the following Euler product

$$
L(\phi, A)=\prod_{f} \frac{[A / f A]_{A}}{[\phi(A / f A)]_{A}},
$$

where the product is over irreducible polynomials of $A_{+}$. Taelman proved [22, §5] that $L(\phi)$ is convergent in $\mathbb{K}_{\infty}$. 
We observe that $[A / f A]_{A}=f$. Assume further that $r_{0} \geq 1$. Let $h$ be an irreducible polynomial in $A_{+}$which is not equal to $f$, and let

$$
P_{\phi}(x):=x^{r_{0}}+p_{r_{0}-1} x^{r_{0}-1}+\cdots+p_{1} x+p_{0} \in A[x]
$$

be the characteristic polynomial of the action of $\tau^{d}$ on the Tate module $T_{h}(\bar{\phi})$ (see [14, $\left.\S 1\right]$ and [16, §4.10] for details about the Tate module). By [8, Cor. 3.2(a)], we know that $\operatorname{deg}_{\theta} p_{i}<d$ for $1 \leq i \leq r_{0}-1$, and $p_{0}=c(f)^{-1} f$ for some $c(f) \in \mathbb{F}_{q}^{\times}$. By the work of Gekeler [14, Thm. 5.1(i)] (see also [8, Cor. 3.2(b)]), we know further that $[\phi(A / f A)]_{A}=c(f) P_{\phi}(1)$.

Define the polynomial $D_{f}^{\phi}(x):=1+c(f) p_{1} x+c(f) p_{2} f x^{2}+\cdots+c(f) f^{r_{0}-1} x^{r_{0}}$. Following [8, §3], for any $s \in \mathbb{Z}$, the dual Goss $L$-series $L\left(\phi^{\vee}, s-1\right)$ is given by

$$
L\left(\phi^{\vee}, s-1\right)=\prod_{f \text { prime in } A_{+}} D_{f}^{\phi}\left(f^{-s}\right)^{-1}=\sum_{a \in A_{+}} \frac{\mu(a)}{a^{s}}
$$

where the function $\mu: A_{+} \rightarrow A$ is defined by the Euler product expansion above and has the generating series (see also $[\underline{8}, \S 3]$ )

$$
\sum_{i=0}^{\infty} \mu\left(f^{i}\right) x^{i}=D_{f}^{\phi}(x)^{-1}
$$

Lemma 2.2 (Chang, El-Guindy, Papanikolas [8, Lem. 3.5]). Let a be an element in $A_{+}$.

(a) The function $\mu: A_{+} \rightarrow A$ is multiplicative.

(b) $\operatorname{deg}_{\theta} \mu(a) \leq\left(1-\frac{1}{r}\right) \operatorname{deg}_{\theta}(a)$.

2.4. Drinfeld $\tilde{A}$-Modules. Throughout this section, we set $z:=z_{1}$, and abbreviate $\mathbb{T}_{1}$ by $\mathbb{T}$. We also fix the notation $\tilde{A}$ for $\mathbb{F}_{q}(z)[\theta]$. Let $m \geq 1$ be an integer. Inspired by [6, §2.4], we define the $z^{m}$-deformation of Drinfeld $A$-module $\phi$ as an $\mathbb{F}_{q}(z)$-algebra homomorphism $\tilde{\phi}: \tilde{A} \rightarrow \tilde{A}\{\tau\}$ given by

$$
\tilde{\phi}_{\theta}=\sum_{i=0}^{r} \tilde{\phi}_{\theta, i} \tau^{i}=\sum_{i=0}^{r} z^{m i} \phi_{\theta, i} \tau^{i}
$$

Note that if $r_{0}=0$, we have that $\tilde{\phi}_{f, j} \equiv 0(\bmod f)$ for all $j \geq 0$.

Lemma 2.3. Let $f$ be an irreducible polynomial in $A_{+}$of degree $d$ with $r_{0} \geq 1$, and let $\tilde{\phi}_{f, k}$ be the $k^{\text {th }}$ coefficient of $\tilde{\phi}_{f}$. Then for all $0 \leq j \leq d-1$, we have

$$
\tilde{\phi}_{f, j} \equiv 0 \quad(\bmod f)
$$

and for $d \leq j \leq r_{0} d$, we have

$$
\tilde{\phi}_{f, j} \equiv \sum_{i=1}^{\lfloor j / d\rfloor} z^{m i d} \tilde{\phi}_{-c(f) p_{i}, j-i d} \quad(\bmod f)
$$

where $\lfloor\cdot\rfloor$ is the floor function.

Proof. Observe that for any $k \geq 0$, we have $\tilde{\phi}_{f, k}=z^{m k} \phi_{f, k}$. By [8, Lem. 5.4(a)], for $0 \leq j \leq$ $d-1$, we get

$$
\phi_{f, j} \equiv 0 \quad(\bmod f) .
$$


Thus, the first part follows from (10). By [8, Lem. 5.4(b)], we see that for $d \leq j \leq r_{0} d$,

$$
\phi_{f, j} \equiv \sum_{i=1}^{\lfloor j / d\rfloor} \phi_{-c(f) p_{i}, j-i d}(\bmod f) \text {. }
$$

Therefore,

$$
\tilde{\phi}_{f, j}=z^{m j} \phi_{f, j} \equiv \sum_{i=1}^{\lfloor j / d\rfloor} z^{m i d} z^{m j-m i d} \phi_{-c(f) p_{i}, j-i d} \equiv \sum_{i=1}^{\lfloor j / d\rfloor} z^{m i d} \tilde{\phi}_{-c(f) p_{i}, j-i d} \quad(\bmod f) .
$$
that

$$
[\tilde{\phi}(\tilde{A} / f \tilde{A})]_{\tilde{A}}=\operatorname{det}_{\mathbb{F}_{q}(z)[X]}\left(X-\tilde{\phi}_{\theta} \mid \tilde{A} / f \tilde{A} \otimes_{\mathbb{F}_{q}(z)} \mathbb{F}_{q}(z)[X]\right)_{\mid X=\theta}
$$

In other words, $[\tilde{\phi}(\tilde{A} / f \tilde{A})]_{\tilde{A}}$ is the evaluation at $X=\theta$ of the characteristic polynomial of the action of $\theta$ on the $\tilde{A}$-module $\tilde{A} / f \tilde{A} \otimes_{\mathbb{F}_{q}(z)} \mathbb{F}_{q}(z)$. The monic generator $[\tilde{\phi}(\tilde{A} / f \tilde{A})]_{\tilde{A}}$ of the Fitting ideal of $\tilde{\phi}(\tilde{A} / f \tilde{A})$ is an element in $\tilde{A}$. By definition, it is a polynomial in $\theta$ of degree $d$, and is a polynomial in $z^{m}$ of degree at most $r_{0} d$. One can observe that if $r_{0}=0$, then

$$
[\tilde{\phi}(\tilde{A} / f \tilde{A})]_{\tilde{A}}=[\tilde{A} / f \tilde{A}]_{\tilde{A}}=f .
$$

Proposition 2.4. For any irreducible polynomial $f \in A_{+}$of degree d with $r_{0} \geq 1$, we have

$$
[\tilde{\phi}(\tilde{A} / f \tilde{A})]_{\tilde{A}}=f+c(f) p_{1} z^{m d}+c(f) p_{2} z^{2 m d}+\cdots+c(f) z^{r_{0} m d} .
$$

Proof. To ease the notation, let us define $Q(z):=[\tilde{\phi}(\tilde{A} / f \tilde{A})]_{\tilde{A}}$. Note that if we set $z=0$ in (9), then $\tilde{\phi}$ becomes the trivial Drinfeld $A$-module meaning that the $A$-module action on $\tilde{\phi}(\tilde{A} / f \tilde{A})$ is given by multiplying elements in $A / f A$ by $\theta$. Therefore, by (12), we have that $Q(0)=f$. Now let $A_{i} \in A$ for $1 \leq i \leq r_{0} d$, and set $Q(z)=f+A_{1} z^{m}+A_{2} z^{2 m}+\cdots+A_{r_{0} d} z^{r_{0} m d}$. Since $Q(z)$ is a monic polynomial in $\theta$ of degree $d$, we have that $\operatorname{deg}_{\theta}\left(A_{i}\right)<d$ for all $i$. On the other hand, considering $\tilde{A}$-action on the module $\tilde{A} / f \tilde{A}$ shows that all elements in $\tilde{A} / f \tilde{A}$ is annihilated by $Q(z)$. That is, for any $x \in \tilde{A} / f \tilde{A}$, we have by Lemma 2.3 that

$$
\begin{aligned}
Q(z) \cdot x & =z^{m} \tilde{\phi}_{A_{1}}(x)+\cdots+z^{m d} \tilde{\phi}_{A_{d}}(x)+\cdots+z^{r_{0} m d} \tilde{\phi}_{A_{r_{0} d}}(x)+\tilde{\phi}_{f}(x) \\
& \equiv z^{m} \tilde{\phi}_{A_{1}}(x)+\cdots+z^{m d} \tilde{\phi}_{A_{d}}(x)+\cdots+z^{r_{0} m d} \tilde{\phi}_{A_{r_{0}}}(x)+\sum_{j=d}^{r_{0} d} \sum_{i=1}^{\lfloor j / d\rfloor} z^{m i d} \tilde{\phi}_{-c(f) p_{i}, j-i d}(x) \\
& \equiv 0 \quad(\bmod f) .
\end{aligned}
$$

Since $\operatorname{deg}_{\theta}\left(A_{i}\right)<d$ for all $i$, we have that $z^{m} \tilde{\phi}_{A_{1}, 0}(x)=z^{m} A_{1} x=0$. This implies that $A_{1}=0$. By induction, we see that $A_{i}=0$ for all $1 \leq i \leq d-1$. Now, the coefficient of $z^{m d}$ term in $Q(z) \cdot x$ becomes $\left(\tilde{\phi}_{-c(f) p_{1}, 0}+\tilde{\phi}_{A_{d, 0}}\right) x$. But this term is equal to zero for all $x \in \tilde{A} / f \tilde{A}$. Thus, we have $\tilde{\phi}_{-c(f) p_{1}, 0}=-\tilde{\phi}_{A_{d, 0}}$ by the fact the degrees of $p_{1}$ and $A_{d}$ are less than $d$. Therefore, we have $c(f) p_{1}=A_{d}$. Moreover, the coefficient of $z^{m(d+1)}$ term in $Q(z) \cdot x$ appears as $\left(\tilde{\phi}_{-c(f) p_{1}, 1}+\tilde{\phi}_{A_{d, 1}}\right) x^{q}+\tilde{\phi}_{A_{d+1,0}} x$. Notice that the first two terms cancel out, and the expression is equal to 0 for all $x$. This implies that $A_{d+1}=0$. Similar calculation as above 
shows that $A_{d+j}=0$ for all $1 \leq j \leq d-1$. If we apply the same logic to other coefficients of $Q(z)$, we see that $Q(z)=f+c(f) p_{1} z^{m d}+c(f) p_{2} z^{2 m d}+\cdots+c(f) z^{r_{0} m d}$ as desired.

Remark 2.5. (See also proof of [5, Prop. 6.2].) Let $f$ be an irreducible polynomial in $A_{+}$ defined by $f=a_{0}+a_{1} \theta+a_{2} \theta^{2}+\cdots+\theta^{d}$ such that $r_{0} \geq 1$. Let $\overline{\mathbb{F}}_{q}$ be the algebraic closure of $\mathbb{F}_{q}$. Since the characteristic polynomial is invariant under the extension of scalars, without loss of generality, we can replace the field $\tilde{A} / f \tilde{A} \otimes_{\mathbb{F}_{q}(z)} \mathbb{F}_{q}(z)$ in (12) by $F=\tilde{A} / f \tilde{A} \otimes_{\mathbb{F}_{q}(z)} \overline{\mathbb{F}}_{q}(z)$.

Let $\operatorname{Gal}\left(\tilde{A} / f \tilde{A} / \mathbb{F}_{q}(z)\right)$ be the Galois group of the field extension $\tilde{A} / f \tilde{A}$ of $\mathbb{F}_{q}(z)$. We have the isomorphism

$$
F \cong \prod_{g \in \operatorname{Gal}\left(\tilde{A} / f \tilde{A} / \mathbb{F}_{q}(z)\right)} \overline{\mathbb{F}}_{q}(z)
$$

via a map sending $a \otimes b \in F$ to $(g(a) b)_{g \in S}$ where $S$ is the set of embeddings of $\tilde{A} / f \tilde{A}$ into $\overline{\mathbb{F}}_{q}(z)$. Observe that $\left\{1 \otimes 1, \bar{\theta} \otimes 1, \bar{\theta}^{2} \otimes 1, \ldots, \bar{\theta}^{d-1} \otimes 1\right\}$ is an $\overline{\mathbb{F}}_{q}(z)$-basis for $F$. Furthermore, we have that

$$
\theta \cdot\left[\begin{array}{c}
1 \otimes 1 \\
\bar{\theta} \otimes 1 \\
\vdots \\
\vdots \\
\bar{\theta}^{d-1} \otimes 1
\end{array}\right]=P\left[\begin{array}{c}
1 \otimes 1 \\
\bar{\theta} \otimes 1 \\
\vdots \\
\vdots \\
\bar{\theta}^{d-1} \otimes 1
\end{array}\right]=\left[\begin{array}{ccccc}
0 & 1 & 0 & \ldots & 0 \\
0 & 0 & 1 & \ddots & \vdots \\
\vdots & \vdots & \ddots & \ddots & 0 \\
\vdots & \vdots & \vdots & 0 & 1 \\
-a_{0} & -a_{1} & \ldots & \ldots & -a_{d-1}
\end{array}\right]\left[\begin{array}{c}
1 \otimes 1 \\
\bar{\theta} \otimes 1 \\
\vdots \\
\vdots \\
\bar{\theta}^{d-1} \otimes 1
\end{array}\right]
$$

where $P$ is the transpose of a companion matrix defined above. Let $\eta \in \overline{\mathbb{F}}_{q}$ be a fixed root of $f$. Then, there exists $Q \in \mathrm{GL}_{d}\left(\overline{\mathbb{F}}_{q}\right)$ such that

$$
Q P Q^{-1}=\left[\begin{array}{ccccc}
\eta & 0 & 0 & 0 & 0 \\
0 & \eta^{q} & 0 & \ddots & 0 \\
0 & 0 & \ddots & \ddots & \vdots \\
\vdots & \vdots & \ddots & \ddots & 0 \\
0 & 0 & 0 & 0 & \eta^{q^{d-1}}
\end{array}\right]
$$

Now, define $\left\{v_{1}, v_{2}, \ldots, v_{d}\right\}$ by

$$
\left[\begin{array}{c}
v_{1} \\
v_{2} \\
\vdots \\
v_{d}
\end{array}\right]=Q\left[\begin{array}{c}
1 \otimes 1 \\
\bar{\theta} \otimes 1 \\
\vdots \\
\bar{\theta}^{d-1} \otimes 1
\end{array}\right]
$$

Therefore, the set $\left\{v_{1}, \ldots, v_{d}\right\}$ is an $\overline{\mathbb{F}}_{q}(z)$-basis for $F$ and $\overline{\mathbb{F}}_{q}(z)[X]$-basis for $F[X]$. Moreover, considering indices modulo $d$, we have $\theta \cdot v_{i}=m_{i} v_{i}$ where $m_{i} \in \overline{\mathbb{F}}_{q}^{\times}$such that $f(z)=$ $\prod_{i=1}^{d}\left(z-m_{i}\right)$, and $m_{i}^{q}=m_{i+1}$. Furthermore, we have that

$$
\tau \cdot v_{i}=v_{i-1} .
$$


Thus, for any basis element $v_{j}$, we have that

$$
\begin{aligned}
& \left(X-\tilde{\phi}_{\theta}\right) \cdot v_{j}=\left(X-\sum_{k=0}^{r_{0}} z^{m k} \phi_{\theta, k} \tau^{k}\right) \cdot v_{j} \\
& =\left(X-m_{j}-\sum_{i \geq 1, r_{0}-d i \geq 0} z^{m d i} \phi_{\theta, d i}\left(m_{j}\right)\right) v_{j}+\left(-\phi_{\theta, 1}\left(m_{j-1}\right) z^{m}-\sum_{i \geq 1, r_{0}-d i \geq 1} z^{m d i+m} \phi_{\theta, d i+1}\left(m_{j-1}\right)\right) v_{j-1} \\
& +\cdots+\left(-\phi_{\theta, d-1}\left(m_{j-(d-1)}\right) z^{m d-m}-\sum_{i \geq 1, r_{0}-d i \geq d-1} z^{m d i+m d-m} \phi_{\theta, d i+d-1}\left(m_{j-(d-1)}\right)\right) v_{j-(d-1)} .
\end{aligned}
$$

\section{TAELMAN $L$-VAlues}

In this section, we analyze Taelman $L$-values corresponding to special Drinfeld modules over Tate algebras which were introduced by Anglès and Tavares Ribeiro in [6, §3]. For more information about Drinfeld modules over Tate algebras, we direct the reader to [3] and [15].

3.1. Drinfeld $\mathbb{A}$-Modules. In this section, we investigate the Taelman $L$-value corresponding to the Drinfeld $\mathbb{A}$-module $\varphi$ defined in (2).

We note that the Drinfeld $\mathbb{A}$-module $\varphi$ has an exponential function $\exp _{\varphi}: \widetilde{\mathbb{T}_{n}} \rightarrow \widetilde{\mathbb{T}_{n}}$ which is defined by

$$
\exp _{\varphi}(g)=\sum_{j} \ell_{j}\left(z_{1}\right) \ldots \ell_{j}\left(z_{n}\right) \alpha_{j} \tau^{j}(g)
$$

for all $g \in \widetilde{\mathbb{T}_{n}}$ where $\alpha_{j}$ 's are coefficients of $\exp _{\phi}$. Note that by [15, Prop. 3.2.3], $\exp _{\varphi}$ converges everywhere on $\mathbb{T}_{n}$. Its logarithm function $\log _{\varphi}: \widetilde{\mathbb{T}_{n}} \rightarrow \widetilde{\mathbb{T}_{n}}$ is given by

$$
\log _{\varphi}(g)=\sum_{j} \ell_{j}\left(z_{1}\right) \ldots \ell_{j}\left(z_{n}\right) \gamma_{j} \tau^{j}(g)
$$

for all $g \in \widetilde{\mathbb{T}_{n}}$ where $\gamma_{j}$ 's are coefficients of $\log _{\phi}$ and $g$ within the radius of convergence of $\log _{\varphi}$. Let $f$ be an irreducible element in $A_{+}$, and let $\varphi(\mathbb{A} / f \mathbb{A})$ be the $\mathbb{A}$-module $\mathbb{A} / f \mathbb{A}$ with the induced action of $\varphi$ on $\mathbb{A} / f \mathbb{A}$. Note that

$$
[\mathbb{A} / f \mathbb{A}]_{\mathbb{A}}=f
$$

and

$$
[\varphi(\mathbb{A} / f \mathbb{A})]_{\mathbb{A}}=\operatorname{det}_{\mathbb{F}_{q}\left(z_{1}, \ldots, z_{n}\right)[X]}\left(X-\varphi_{\theta} \mid \mathbb{A} / f \mathbb{A} \otimes_{\mathbb{F}_{q}\left(z_{1}, \ldots, z_{n}\right)} \mathbb{F}_{q}\left(z_{1}, \ldots, z_{n}\right)[X]\right)_{\mid X=\theta} .
$$

The Taelman $L$-value $L(\varphi, \mathbb{A})$ corresponding to $\varphi$ is defined by the following Euler product

$$
L(\varphi, \mathbb{A})=\prod_{f} \frac{[\mathbb{A} / f \mathbb{A}]_{\mathbb{A}}}{[\varphi(\mathbb{A} / f \mathbb{A})]_{\mathbb{A}}},
$$

where the product is over irreducible polynomials of $A_{+}$. By [9, Thm. 2.7] (see also [6, §3.1]), $L(\varphi, \mathbb{A})$ converges in the ring $\mathbb{F}_{q}\left(z_{1}, \ldots, z_{n}\right)\left(\left(\theta^{-1}\right)\right)$. 
Remark 3.1. Let $f$ be a prime in $A_{+}$of degree $d$ with $r_{0} \geq 1$. As in Remark 2.5, since the characteristic polynomial is invariant under the extension of scalars, without loss of generality, we can replace the ring $\mathbb{A} / f \mathbb{A} \otimes_{\mathbb{F}_{q}\left(z_{1}, \ldots, z_{n}\right)} \mathbb{F}_{q}\left(z_{1}, \ldots, z_{n}\right)$ in (17) by the ring

$$
F=\mathbb{A} / f \mathbb{A} \otimes_{\mathbb{F}_{q}\left(z_{1}, \ldots, z_{n}\right)} \overline{\mathbb{F}}_{q}\left(z_{1}, \ldots, z_{n}\right) \cong \prod_{g \in \operatorname{Gal}\left(\mathbb{A} / f \mathbb{A} / \mathbb{F}_{q}\left(z_{1}, \ldots, z_{n}\right)\right)} \overline{\mathbb{F}}_{q}\left(z_{1}, \ldots, z_{n}\right) .
$$

Moreover, by the similar calculations in Remark 2.5, we can pick the $\overline{\mathbb{F}}_{q}\left(z_{1}, \ldots, z_{n}\right)$-basis $\left\{v_{1}, \ldots, v_{n}\right\}$ for $F$ such that (14) holds. Furthermore, $\theta \cdot v_{i}=m_{i} v_{i}$ where $m_{i} \in \overline{\mathbb{F}}_{q}^{\times}$with the property that for $1 \leq k \leq n$,

$$
f\left(z_{k}\right)=\prod_{i=1}^{d}\left(z_{k}-m_{i}\right)
$$

and $m_{i}^{q}=m_{i+1}$ so that the indices are modulo $d$. For $1 \leq k \leq n$ and $1 \leq i \leq d$, let us define

$$
t_{k, i}=z_{k}-m_{i} .
$$

Thus, for any basis element $v_{j}$, we have that

$$
\begin{aligned}
& \left(X-\varphi_{\theta}\right) \cdot v_{j}=\left(X-\sum_{i=0}^{r_{0}} \ell_{i}\left(z_{1}\right) \ldots \ell_{i}\left(z_{n}\right) \phi_{\theta, i} \tau^{i}\right) \cdot v_{j} \\
& =\left(X-m_{j}-\sum_{i \geq 1, r_{0}-d i \geq 0} \phi_{\theta, d i}\left(m_{j}\right) \prod_{k=1}^{n} f\left(z_{k}\right)^{i}\right) v_{j}+ \\
& \left(-\phi_{\theta, 1}\left(m_{j-1}\right) \prod_{k=1}^{n} t_{k, j-1}-\sum_{i \geq 1, r_{0}-d i \geq 1} \phi_{\theta, d i+1}\left(m_{j-1}\right) \prod_{k=1}^{n} t_{k, j-1} f\left(z_{k}\right)^{i}\right) v_{j-1}+\ldots \\
& +\left(-\phi_{\theta, d-1}\left(m_{j-(d-1)}\right) \prod_{k=1}^{n} t_{k, j-(d-1)} \ldots t_{k, j-1}\right. \\
& \left.-\sum_{i \geq 1, r_{0}-d i \geq d-1} \phi_{\theta, d i+d-1}\left(m_{j-(d-1)}\right) \prod_{k=1}^{n} t_{k, j-(d-1)} \ldots t_{k, j-1} f\left(z_{k}\right)^{i}\right) v_{j-(d-1)} .
\end{aligned}
$$

We now recall the definition of $r_{0}$ from $\S 2.3$ and observe that if $r_{0}=0$, then

$$
[\varphi(\mathbb{A} / f \mathbb{A})]_{\mathbb{A}}=[\mathbb{A} / f \mathbb{A}]_{\mathbb{A}}=f
$$

Proposition 3.2. Let $\varphi$ be a Drinfeld $\mathbb{A}$-module of rank $r$ defined in (2). Then for all irreducible $f \in A_{+}$of degree d with $r_{0} \geq 1$, we have

$$
[\varphi(\mathbb{A} / f \mathbb{A})]_{\mathbb{A}}=f+c(f) p_{1} \prod_{k=1}^{n} f\left(z_{k}\right)+c(f) p_{2} \prod_{k=1}^{n} f\left(z_{k}\right)^{2}+\cdots+c(f) \prod_{k=1}^{n} f\left(z_{k}\right)^{r_{0}} .
$$

Proof. We extend the idea of Anglès and Taelman in the proof of [5, Prop. 6.2]. Assume that $n=1$, and fix an irreducible polynomial $f \in A_{+}$of degree $d$ with $r_{0} \geq 1$. Set $t_{i}:=t_{1, i}$. We see from (20) and (21) that

$$
f\left(z_{1}\right)=\prod_{i=1}^{d} t_{i}
$$


Let $F=\mathbb{A} / f \mathbb{A} \otimes_{\mathbb{F}_{q}\left(z_{1}\right)} \overline{\mathbb{F}}_{q}\left(z_{1}\right)$ and $R_{\varphi}$ be the matrix representing the map $X-\varphi_{\theta}$ on $F[X]$ with respect to the $\overline{\mathbb{F}}_{q}\left(z_{1}\right)[X]$-basis $\left\{v_{1}, \ldots, v_{d}\right\}$. We claim that $\operatorname{det}\left(R_{\varphi}\right)$ is a polynomial in $f\left(z_{1}\right)$ which has constant coefficient equal to $f(X)$. By (22), it is enough to look at $\operatorname{det}\left(R_{\varphi}\right)$ when $r_{0}<d$. Let $R$ be the matrix defined by

$$
R=\left[a_{i, j}\right]:=\left[\begin{array}{cccc}
X-m_{1} & -\phi_{\theta, 1}\left(m_{1}\right) & \ldots & -\phi_{\theta, d-1}\left(m_{1}\right) \\
-\phi_{\theta, d-1}\left(m_{2}\right) & X-m_{2} & \ldots & \vdots \\
\vdots & -\phi_{\theta, d-1}\left(m_{3}\right) & \ldots & \vdots \\
\vdots & \vdots & \ldots & -\phi_{\theta, 2}\left(m_{d-2}\right) \\
-\phi_{\theta, 2}\left(m_{d-1}\right) & \vdots & \ldots & -\phi_{\theta, 1}\left(m_{d-1}\right) \\
-\phi_{\theta, 1}\left(m_{d}\right) & -\phi_{\theta, 2}\left(m_{d}\right) & \ldots & X-m_{d}
\end{array}\right]
$$

and set

$$
b_{i, j}:= \begin{cases}a_{i j} t_{i} t_{i+1} \ldots t_{j-1+d} & \text { if } i>j \\ a_{i j} \prod_{k=i}^{j-1} t_{k} & \text { if } i<j \\ a_{i j} & \text { if } i=j\end{cases}
$$

By (22), one can show that $R_{\varphi}=\left[b_{i, j}\right]$ when $r_{0}<d$. Let $S_{d}$ be the symmetric group of degree $d$ and $\operatorname{sgn}(\sigma)$ be the sign of a permutation $\sigma \in S_{d}$. Then by Leibniz formula, we have

$$
\operatorname{det}\left(R_{\varphi}\right)=\sum_{\sigma \in S_{d}} \operatorname{sgn}(\sigma)\left(\prod_{i=1}^{d} b_{\sigma(i), i}\right) .
$$

Notice that

$$
f(X)=\prod_{i=1}^{d}\left(X-m_{i}\right)=\prod_{i=1}^{d} b_{i i} .
$$

Thus, the identity permutation corresponds to the term $f(X)$ in $\operatorname{det}\left(R_{\varphi}\right)$. By (24) and (25), we observe that for any permutation $\sigma \in S_{d}$, it is enough to look at images of $1 \leq i \leq d$ which are not fixed by $\sigma$ to prove the claim. Now let $\sigma=\left(i_{1} i_{2} \ldots i_{j}\right)$ be a $j$-cycle in $S_{d}$. We have

$$
\begin{aligned}
\prod_{i \in\left\{i_{1}, \ldots, i_{j}\right\}} b_{\sigma(i), i} & =t_{\sigma\left(i_{j}\right)} t_{\sigma\left(i_{j}\right)+1} \ldots t_{i_{j}-1} t_{\sigma\left(i_{j-1}\right)} t_{\sigma\left(i_{j-1}\right)+1} \ldots t_{i_{j-1}-1} \ldots t_{\sigma\left(i_{1}\right)} \ldots t_{i_{1}-1} \prod_{i \in\left\{i_{1}, \ldots, i_{j}\right\}} a_{\sigma(i), i} \\
& =t_{i_{1}} t_{i_{1}+1} \ldots t_{i_{j}-1} t_{i_{j}} t_{i_{j}+1} \ldots t_{i_{j-1}-1} \ldots t_{i_{2}} \ldots t_{i_{1}-1} \prod_{i \in\left\{i_{1}, \ldots, i_{j}\right\}} a_{\sigma(i), i}
\end{aligned}
$$

so that the indices are modulo $d$. Therefore, we see from (23) and the last line of (27) that there exists a positive integer $k_{\sigma}$ such that

$$
\prod_{i \in\left\{i_{1}, \ldots, i_{j}\right\}} b_{\sigma(i), i}=f\left(z_{1}\right)^{k_{\sigma}} \prod_{i \in\left\{i_{1}, \ldots, i_{j}\right\}} a_{\sigma(i), i}
$$

Thus, the claim follows from (25), (26), and (28) together with the fact that every permutation of $S_{d}$ is a product of disjoint cycles [10, $\left.\S 1.3\right]$. 
Using (15) and (22) after choosing $m=1$, we see that the coefficient of $f\left(z_{1}\right)^{i}$ term in $\operatorname{det}\left(R_{\varphi}\right)$ is the coefficient of $z^{d i}$ term in $[\tilde{\phi}(\tilde{A} / f \tilde{A})]_{\tilde{A}}$. Therefore, by Proposition 2.4, we have that

$$
\operatorname{det}(R)=f(X)+c(f) p_{1}(X) f\left(z_{1}\right)+c(f) p_{2}(X) f\left(z_{1}\right)^{2}+\cdots+c(f) f\left(z_{1}\right)^{r_{0}} .
$$

The proposition follows from evaluating $\operatorname{det}(R)$ at $X=\theta$ when $n=1$.

Now for arbitrary $n$, we replace the field $F$ by $\mathbb{A} / f \mathbb{A} \otimes_{\mathbb{F}_{q}\left(z_{1}, \ldots, z_{n}\right)} \overline{\mathbb{F}}_{q}\left(z_{1}, \ldots, z_{n}\right)$. Then, to finish the proof, we apply the same argument above by choosing $m=n$ in (15) and noticing that the coefficient of $\prod_{k=1}^{n} f\left(z_{k}\right)^{i}$ term in $\operatorname{det}\left(R_{\varphi}\right)$ is the coefficient of $z^{n d i}$ term in $[\tilde{\phi}(\tilde{A} / f \tilde{A})]_{\tilde{A}}$.

Theorem 3.3. Let $\varphi$ be a Drinfeld $\mathbb{A}$-module as in (2), and $L(\varphi, \mathbb{A})$ be the Taelman L-value corresponding to $\varphi$ defined in (18). Then

$$
L(\varphi, \mathbb{A})=\prod_{f} \frac{[\mathbb{A} / f \mathbb{A}]_{\mathbb{A}}}{[\varphi(\mathbb{A} / f \mathbb{A})]_{\mathbb{A}}}=\sum_{a \in A_{+}} \frac{\mu(a) a\left(z_{1}\right) \ldots a\left(z_{n}\right)}{a},
$$

where the product runs over irreducible polynomials $f$ of $A_{+}$.

Proof. For any irreducible polynomial $f \in A_{+}$with $r_{0} \geq 1$, define the polynomial

$$
\begin{aligned}
D_{f}^{\varphi}(x) & :=D_{f}^{\phi}\left(f\left(z_{1}\right) \ldots f\left(z_{n}\right) x\right) \\
& =1+c(f) p_{1} \prod_{i=1}^{n} f\left(z_{i}\right) x+c(f) p_{2} f \prod_{i=1}^{n} f\left(z_{i}\right)^{2} x^{2}+\cdots+c(f) f^{r_{0}-1} \prod_{i=1}^{n} f\left(z_{i}\right)^{r_{0}} x^{r_{0}} .
\end{aligned}
$$

For $s \geq 1$, define the following $L$-series by

$$
L\left(\varphi^{\vee}, s-1\right):=\prod_{f} D_{f}^{\varphi}\left(f^{-s}\right)^{-1}=\sum_{a \in A_{+}} \frac{\mu_{\varphi}(a)}{a^{s}},
$$

where the product is over irreducible polynomials $f$ in $A_{+}$, and $\mu_{\varphi}: A_{+} \rightarrow \mathbb{A}$ is a function satisfying (29). Using (16) and Proposition [3.2, we see that

$$
\begin{aligned}
\frac{[\mathbb{A} / f \mathbb{A}]_{\mathbb{A}}}{[\varphi(\mathbb{A} / f \mathbb{A})]_{\mathbb{A}}} & =\frac{f}{f+c(f) p_{1} \prod_{i=1}^{n} f\left(z_{i}\right)+c(f) p_{2} \prod_{i=1}^{n} f\left(z_{i}\right)^{2}+\cdots+c(f) \prod_{i=1}^{n} f\left(z_{i}\right)^{r_{0}}} \\
& =\frac{1}{1+c(f) p_{1} f^{-1} \prod_{i=1}^{n} f\left(z_{i}\right)+\cdots+c(f) f^{r_{0}-1} f^{-r_{0}} \prod_{i=1}^{n} f\left(z_{i}\right)^{r_{0}}} \\
& =D_{f}^{\varphi}\left(f^{-1}\right)^{-1} .
\end{aligned}
$$

Thus, by (18) and (29),$L(\varphi, \mathbb{A})=L\left(\varphi^{\vee}, 0\right)$. Observe that (8) implies

$$
\sum_{i=0}^{\infty}\left(f\left(z_{1}\right) \ldots f\left(z_{n}\right)\right)^{i} \mu\left(f^{i}\right) x^{i}=D_{f}^{\phi}\left(f\left(z_{1}\right) \ldots f\left(z_{n}\right) x\right)^{-1}=D_{f}^{\varphi}(x)^{-1} .
$$

Since by Lemma 2.2(a), $\mu: A_{+} \rightarrow A$ is a multiplicative function, (30) implies that $\mu_{\varphi}(a)=$ $\mu(a) a\left(z_{1}\right) \ldots a\left(z_{n}\right)$ for all $a \in A_{+}$. Thus,

$$
L(\varphi, \mathbb{A})=L\left(\varphi^{\vee}, 0\right)=\prod_{f \text { irreducible in } A_{+}} D_{f}^{\varphi}\left(f^{-1}\right)^{-1}=\sum_{a \in A_{+}} \frac{\mu(a) a\left(z_{1}\right) \ldots a\left(z_{n}\right)}{a} .
$$


3.2. Drinfeld $\tilde{\mathbb{A}}$-Modules. Let $\phi$ be a Drinfeld $A$-module as in (11) and $t$ be an indeterminate over $\mathbb{C}_{\infty}$. Let $\tilde{\mathbb{A}}$ be the polynomial ring $\mathbb{F}_{q}\left(z_{1}, \ldots, z_{n}, t\right)[\theta]$. We define the Drinfeld $\tilde{\mathbb{A}}$-module $\psi$ as an $\mathbb{F}_{q}\left(z_{1}, \ldots, z_{n}, t\right)$-algebra homomorphism $\psi: \tilde{\mathbb{A}} \rightarrow \tilde{\mathbb{A}}\{\tau\}$ by

$$
\psi_{\theta}=\sum_{i=1}^{r} \psi_{\theta, i} \tau^{i}=\sum_{i=1}^{r} t^{i} \ell_{i}\left(z_{1}\right) \ldots \ell_{i}\left(z_{n}\right) \phi_{\theta, i} \tau^{i}
$$

It has the exponential series $\exp _{\psi}=\sum_{i \geq 0} \alpha_{j} \ell_{j}\left(z_{1}\right) \ldots \ell_{j}\left(z_{n}\right) t^{j} \tau^{j} \in \mathbb{T}_{n, t}[[\tau]]$ which induces the exponential function $\exp _{\psi}: \widetilde{\mathbb{T}_{n, t}} \rightarrow \widetilde{\mathbb{T}_{n, t}}$ that converges on $\mathbb{T}_{n, t}$ by [15, Prop. 3.2.3], and is defined by

$$
\exp _{\psi}(g)=\sum_{j} \ell_{j}\left(z_{1}\right) \ldots \ell_{j}\left(z_{n}\right) t^{j} \alpha_{j} \tau^{j}(g)
$$

for all $g \in \widetilde{\mathbb{T}_{n, t}}$. Similarly, it has the logarithm series $\log _{\psi}=\sum_{i \geq 0} \gamma_{j} \ell_{j}\left(z_{1}\right) \ldots \ell_{j}\left(z_{n}\right) t^{j} \tau^{j} \in$ $\mathbb{T}_{n, t}[[\tau]]$, and it induces the logarithm function $\log _{\psi}: \widetilde{\mathbb{T}_{n, t}} \rightarrow \widetilde{\mathbb{T}_{n, t}}$ which is defined by

$$
\log _{\psi}(g)=\sum_{j} \ell_{j}\left(z_{1}\right) \ldots \ell_{j}\left(z_{n}\right) t^{j} \gamma_{j} \tau^{j}(g)
$$

for all $g \in \widetilde{\mathbb{T}_{n, t}}$ in the domain of $\log _{\psi}$. We construct the Taelman $L$-value $L(\psi, \tilde{\mathbb{A}})$ by

$$
L(\psi, \tilde{\mathbb{A}})=\prod_{f} \frac{[\tilde{\mathbb{A}} / f \tilde{\mathbb{A}}]_{\tilde{\mathbb{A}}}}{[\psi(\tilde{\mathbb{A}} / f \tilde{\mathbb{A}})]_{\tilde{\mathbb{A}}}},
$$

where the product runs over irreducible polynomials $f$ of $A_{+}$. We remark that by $[9$, Thm. 2.7] (see also [6, §3.1]), $L(\psi, \tilde{\mathbb{A}})$ converges in the ring $\mathbb{F}_{q}\left(z_{1}, \ldots, z_{n}, t\right)\left(\left(\theta^{-1}\right)\right)$. We observe that

$$
[\psi(\tilde{\mathbb{A}} / f \tilde{\mathbb{A}})]_{\tilde{\mathbb{A}}}=\operatorname{det}_{\mathbb{F}_{q}\left(z_{1}, \ldots, z_{n}, t\right)[X]}\left(X-\psi_{\theta} \mid \tilde{\mathbb{A}} / f \tilde{\mathbb{A}} \otimes_{\mathbb{F}_{q}\left(z_{1}, \ldots, z_{n}, t\right)} \mathbb{F}_{q}\left(z_{1}, \ldots, z_{n}, t\right)[X]\right)_{\mid X=\theta}
$$

Remark 3.4. Let $f$ be a monic irreducible polynomial in $A$ of degree $d$ with $r_{0} \geq 1$. Following the same idea in Remark 2.5 and 3.1, we can replace the field $\tilde{\mathbb{A}} / f \tilde{\mathbb{A}} \otimes_{\mathbb{F}_{q}\left(z_{1}, \ldots, z_{n}, t\right)}$ $\mathbb{F}_{q}\left(z_{1}, \ldots, z_{n}, t\right)$ in (33) with $F:=\tilde{\mathbb{A}} / f \widetilde{\mathbb{A}} \otimes_{\mathbb{F}_{q}\left(z_{1}, \ldots, z_{n}, t\right)} \overline{\mathbb{F}}_{q}\left(z_{1}, \ldots, z_{n}, t\right)$. Since $t$ is an indeterminate over $\overline{\mathbb{F}}_{q}\left(z_{1}, \ldots, z_{n}\right)$, the $\overline{\mathbb{F}}_{q}\left(z_{1}, \ldots, z_{n}\right)$-basis $\left\{v_{1}, \ldots, v_{d}\right\}$ in Remark 3.1 is also an 
$\overline{\mathbb{F}}_{q}\left(z_{1}, \ldots, z_{n}, t\right)$-basis for $F$ with the same properties. Thus, we conclude that for $1 \leq j \leq d$,

$$
\begin{aligned}
& \left(X-\psi_{\theta}\right) \cdot v_{j}=\left(X-\sum_{i=0}^{r_{0}} \ell_{i}\left(z_{1}\right) \ldots \ell_{i}\left(z_{n}\right) t^{i} \phi_{\theta, i} \tau^{i}\right) \cdot v_{j} \\
& =\left(X-m_{j}-\sum_{i \geq 1, r_{0}-d i \geq 0} \phi_{\theta, d i}\left(m_{j}\right) \prod_{k=1}^{n} t^{d i} f\left(z_{k}\right)^{i}\right) v_{j}+ \\
& \left(-\phi_{\theta, 1}\left(m_{j-1}\right) t \prod_{k=1}^{n} t_{k, j-1}-\sum_{i \geq 1, r_{0}-d i \geq 1} \phi_{\theta, d i+1}\left(m_{j-1}\right) \prod_{k=1}^{n} t_{k, j-1} t^{d i+1} f\left(z_{k}\right)^{i}\right) v_{j-1}+\ldots \\
& +\left(-\phi_{\theta, d-1}\left(m_{j-(d-1)}\right) t^{d-1} \prod_{k=1}^{n} t_{k, j-(d-1)} \ldots t_{k, j-1}\right. \\
& \left.-\sum_{i \geq 1, r_{0}-d i \geq d-1} \phi_{\theta, d i+d-1}\left(m_{j-(d-1)}\right) \prod_{k=1}^{n} t_{k, j-(d-1)} \ldots t_{k, j-1} t^{d i+d-1} f\left(z_{k}\right)^{i}\right) v_{j-(d-1)} .
\end{aligned}
$$

Theorem 3.5. Let $\psi$ be the Drinfeld $\tilde{\mathbb{A}}$-module defined in (31). Then

$$
L(\psi, \tilde{\mathbb{A}})=\sum_{a \in A_{+}} \frac{\mu(a) a\left(z_{1}\right) \ldots a\left(z_{n}\right) t^{\operatorname{deg}_{\theta}(a)}}{a} .
$$

Proof. We do the proof for $n=1$, and the multivariable version follows similarly. Let $f$ be an irreducible in $A_{+}$of degree $d$. One can observe that if $r_{0}=0$, then

$$
[\psi(\tilde{\mathbb{A}} / f \tilde{\mathbb{A}})]_{\tilde{\mathbb{A}}}=[\tilde{\mathbb{A}} / f \tilde{\mathbb{A}}]_{\tilde{\mathbb{A}}}=f .
$$

Assume that $r_{0} \geq 1$. To calculate $[\psi(\tilde{\mathbb{A}} / f \tilde{\mathbb{A}})]_{\tilde{\mathbb{A}}}$, by using Remark 3.4 and the similar idea of the proof of Proposition 3.2, we see that it is enough to evaluate the determinant of the matrix $Q$ defined by

$$
Q:=\left[\begin{array}{cccc}
X-m_{1} & -\phi_{\theta, 1}\left(m_{1}\right) t_{1} t & \ldots & -\phi_{\theta, d-1}\left(m_{1}\right) t_{1} \ldots t_{d-1} t^{d-1} \\
-\phi_{\theta, d-1}\left(m_{2}\right) t_{2} \ldots t_{d} t^{d-1} & X-m_{2} & \ldots & \vdots \\
\vdots & \vdots & \ldots & \vdots \\
\vdots & \vdots & \ldots & \vdots \\
-\phi_{\theta, 2}\left(m_{d-1}\right) t_{d-1} t_{d} t^{2} & \vdots & \ldots & -\phi_{\theta, 1}\left(m_{d-1}\right) t_{d-1} t \\
-\phi_{\theta, 1}\left(m_{d}\right) t_{d} t & -\phi_{\theta, 2}\left(m_{d}\right) t_{d} t_{1} t^{2} & \ldots & X-m_{d}
\end{array}\right]
$$

where $t_{1, i}=t_{i}$. Using the same argument in the proof of Proposition 3.2, we see that

$$
\begin{aligned}
{[\psi(\tilde{\mathbb{A}} / f \tilde{\mathbb{A}})]_{\tilde{\mathbb{A}}} } & =\operatorname{det}(Q) \\
& =f+c(f) p_{1} t^{d} f\left(z_{1}\right)+c(f) p_{2} t^{2 d} f\left(z_{1}\right)^{2}+\cdots+c(f) t^{r_{0} d} f\left(z_{1}\right)^{r_{0}} .
\end{aligned}
$$

Now let us define

$$
\begin{aligned}
D_{f}^{\psi}(x): & =D_{f}^{\phi}\left(t^{d} f\left(z_{1}\right) x\right) \\
& =1+c(f) p_{1} t^{d} f\left(z_{1}\right) x+c(f) p_{2} t^{2 d} f\left(z_{1}\right)^{2} f x^{2}+\cdots+c(f) t^{d r_{0}} f\left(z_{1}\right)^{d r_{0}} f^{r_{0}-1} x^{r_{0}},
\end{aligned}
$$


and for $s \geq 1$, define

$$
L\left(\psi^{\vee}, s-1\right):=\prod_{f} D_{f}^{\psi}\left(f^{-s}\right)^{-1}=\sum_{a \in A_{+}} \frac{\mu_{\psi}(a)}{a^{s}},
$$

where $f$ runs over primes of $A_{+}$and $\mu_{\psi}: A_{+} \rightarrow \tilde{\mathbb{A}}$ is a function satisfying (35). We have

$$
\begin{aligned}
\frac{[\tilde{\mathbb{A}} / f \tilde{\mathbb{A}}]_{\tilde{\mathbb{A}}}}{[\psi(\tilde{\mathbb{A}} / f \tilde{\mathbb{A}})]_{\tilde{\mathbb{A}}}} & =\frac{f}{f+c(f) p_{1} t^{d} f\left(z_{1}\right)+c(f) p_{2} t^{2 d} f\left(z_{1}\right)^{2}+\cdots+c(f) t^{d r_{0}} f\left(z_{1}\right)^{r_{0}}} \\
& =\frac{1}{1+c(f) p_{1} t^{d} f\left(z_{1}\right) f^{-1}+\cdots+c(f) t^{r_{0} d} f^{r_{0}-1} f\left(z_{1}\right)^{r_{0}} f^{-r_{0}}} \\
& =D_{f}^{\psi}\left(f^{-1}\right)^{-1} .
\end{aligned}
$$

Thus, by (32), $L(\psi, \tilde{\mathbb{A}})=L\left(\psi^{\vee}, 0\right)$. Note that by (8) , we get

$$
\sum_{i=0}^{\infty}\left(f\left(z_{1}\right) t^{d}\right)^{i} \mu\left(f^{i}\right) x^{i}=D_{f}^{\phi}\left(f\left(z_{1}\right) t^{d} x\right)^{-1}=D_{f}^{\psi}(x)^{-1} .
$$

Therefore, Lemma 2.2(a) implies that $\mu_{\psi}(a)=a\left(z_{1}\right) t^{\operatorname{deg}_{\theta}(a)} \mu(a)$ for all $a \in A_{+}$and that

$$
L(\psi, \tilde{\mathbb{A}})=L\left(\psi^{\vee}, 0\right)=\sum_{a \in A_{+}} \frac{\mu(a) a\left(z_{1}\right) t^{\operatorname{deg}_{\theta}(a)}}{a} .
$$

Combining Theorem 3.5 with the result of Anglès and Tavares Ribeiro [6, Prop. 5], we deduce the following proposition.

Proposition 3.6. We have

$$
\exp _{\psi}(L(\psi, \tilde{\mathbb{A}}))=\exp _{\psi}\left(\sum_{a \in A_{+}} \frac{\mu(a) a\left(z_{1}\right) \ldots a\left(z_{n}\right) t^{\operatorname{deg}_{\theta}(a)}}{a}\right) \in A\left[z_{1}, \ldots, z_{n}, t\right] .
$$

4. $L$-SERIES $L\left(\phi^{\vee}, z_{1}, \ldots, z_{n} ; x, y\right)$

We consider the topological group $\mathbb{S}_{\infty}:=\mathbb{C}_{\infty}^{\times} \times \mathbb{Z}_{p}$ where the group action is given by addition componentwise. For any element $a \in A$, we set

$$
\langle a\rangle:=a \theta^{-\operatorname{deg}_{\theta}(a)} \in 1+\frac{1}{\theta} \mathbb{F}_{q}\left[\frac{1}{\theta}\right] .
$$

For any $y \in \mathbb{Z}_{p}$, we define the exponentiation of $\langle a\rangle$ by

$$
\langle a\rangle^{y}:=\sum_{i \geq 0}\left(\begin{array}{l}
y \\
i
\end{array}\right)(\langle a\rangle-1)^{i}
$$

where the binomial $\left(\begin{array}{l}y \\ i\end{array}\right)$ is defined by the Lucas' formula (See [13, Thm. 1]). In particular, as one can also see in [7, $\S 7]$, if the $p$-adic expansion of $y$ is $\sum \beta_{j} p^{j}$ where $\beta_{j} \in\{0,1, \ldots, p-1\}$, and the $p$-adic expansion of $i$ is $\sum_{k=0}^{n} i_{k} p^{k}$ where $i_{k} \in\{0,1, \ldots, p-1\}$, then we define

$$
\left(\begin{array}{l}
y \\
i
\end{array}\right):=\prod_{j=0}^{n}\left(\begin{array}{l}
\beta_{j} \\
i_{j}
\end{array}\right) \text {. }
$$


We also remark that since $\langle a\rangle$ is a 1 -unit for any $a \in A,\langle a\rangle^{y}$ converges in $\mathbb{K}_{\infty}$ for any $y \in \mathbb{Z}_{p}$.

Let $\phi$ be a Drinfeld $A$-module of rank $r$ defined as in (1). For any $(x, y) \in \mathbb{S}_{\infty}$, consider the series

$$
L\left(\phi^{\vee}, z_{1}, \ldots, z_{n} ; x, y\right):=\sum_{d \geq 0} \mathscr{L}_{d, n}(x, y)\left(z_{1}, \ldots, z_{n}\right)
$$

where we set

$$
\mathscr{L}_{d, n}(x, y)\left(z_{1}, \ldots, z_{n}\right):=x^{-d} \sum_{a \in A_{+, d}} \mu(a) a\left(z_{1}\right) \ldots a\left(z_{n}\right)\langle a\rangle^{y}
$$

For any integer $s>0$, we construct the following $L$-Series by

$$
L\left(\phi^{\vee}, z_{1}, \ldots, z_{n}, s\right):=L\left(\phi^{\vee}, z_{1}, \ldots, z_{n} ; \theta^{s},-s\right)=\sum_{a \in A_{+}} \frac{\mu(a) a\left(z_{1}\right) \ldots a\left(z_{n}\right)}{a^{s}} .
$$

Note that by Lemma 2.2(b), $L\left(\phi^{\vee}, z_{1}, \ldots, z_{n}, s\right)$ converges in $\mathbb{T}_{n}$ for any integer $s \geq 1$.

4.1. The Value of $L\left(\phi^{\vee}, z_{1}, \ldots, z_{n}, s\right)$ at $s=1$. Let $\phi$ be a Drinfeld $A$-module of rank $r$ as in (11), $\varphi$ be the Drinfeld $\mathbb{A}$-module as in (21) and $\psi$ be the Drinfeld $\tilde{\mathbb{A}}$-module defined as in (31). Recall that $\beta=\max \left\{\operatorname{deg}_{\theta}\left(\phi_{\theta, i}\right) \mid 1 \leq i \leq r\right\}$. By Theorem 3.3, we have

$$
L\left(\phi^{\vee}, z_{1}, \ldots, z_{n}, 1\right)=L(\varphi, \mathbb{A}) .
$$

In this section, our aim is to relate the Taelman $L$-value $L(\varphi, \mathbb{A})$ to the logarithm function $\log _{\varphi}$. First, we need a proposition.

Proposition 4.1. If $0 \leq n \leq q / r-(1+2 \beta)$, then

$$
\exp _{\psi}\left(\sum_{a \in A_{+}} \frac{\mu(a) a\left(z_{1}\right) \ldots a\left(z_{n}\right) t^{\operatorname{deg}_{\theta}(a)}}{a}\right)=1 .
$$

In particular,

$$
\exp _{\varphi}\left(\sum_{a \in A_{+}} \frac{\mu(a) a\left(z_{1}\right) \ldots a\left(z_{n}\right)}{a}\right)=1 \text {. }
$$

Proof. We adapt the ideas in [6, Lem. 7]. Let $\alpha_{j}$ and $\alpha_{j}^{\prime}$ be the coefficients of $\exp _{\phi}$ and $\exp _{\psi}$ respectively. By [11, Eq. 28], we have for all $j \geq 1$ that

$$
\operatorname{ord}_{\infty}\left(\alpha_{j}\right) \geq q^{j}\left(\frac{j}{r}-\frac{\beta}{q-1}\right)
$$

This means that

$$
\operatorname{ord}\left(\alpha_{j}^{\prime}\right)=\operatorname{ord}\left(\alpha_{j} \ell_{j}\left(z_{1}\right) \ldots \ell_{j}\left(z_{n}\right) t^{j}\right) \geq q^{j}\left(\frac{j}{r}-\frac{\beta+n}{q-1}\right)+\frac{n}{q-1} .
$$

Let $h: \mathbb{R}_{\geq 1} \rightarrow \mathbb{R}$ be a function defined by

$$
h(x)=q^{x}\left(\frac{x}{r}-\frac{\beta+n}{q-1}\right)+\frac{n}{q-1} .
$$


Note that $h$ is an increasing function on $\mathbb{R}_{\geq 1}$ when $\beta+n \leq(q-1) / r$. Moreover, if $\beta+n \leq$ $-1+(n+1) / q+(q-1) / r$ and $q>n$, then for all $x \geq 1$, we have

$$
\begin{aligned}
h(x)=q^{x}\left(\frac{x}{r}-\frac{\beta+n}{q-1}\right)+\frac{n}{q-1} & \geq q\left(\frac{1}{r}-\frac{\beta+n}{q-1}\right)+\frac{n}{q-1} \\
& \geq q\left(\frac{1}{r}+\frac{1}{q-1}-\frac{n+1}{q(q-1)}-\frac{1}{r}\right)+\frac{n}{q-1} \\
& =1 .
\end{aligned}
$$

Since $q \geq 2$ and $r \geq 1$, if $0 \leq n \leq q / r-(1+2 \beta)$, we note that

$$
\begin{aligned}
n \leq \frac{q}{r}-(1+2 \beta) & \Longleftrightarrow q-(r n+r+\beta r) \geq \beta r \\
& \Longleftrightarrow(q-(r n+r+\beta r))(q-1)-\beta r \geq 0 \\
& \Longleftrightarrow q^{2}-q(r n+r+\beta r+1)+r(n+1) \geq 0 \\
& \Longleftrightarrow-1+\frac{n+1}{q}+\frac{q-1}{r} \geq \beta+n,
\end{aligned}
$$

and $q>n$ so that (39) follows. Thus, (38) and (39) imply that $\operatorname{ord}\left(\alpha_{j}^{\prime}\right) \geq 1$ for all $j \geq 1$. On the other hand, by Lemma 2.2(b), we know that

$$
\operatorname{ord}\left(\sum_{a \in A_{+}} \frac{\mu(a) a\left(z_{1}\right) \ldots a\left(z_{n}\right) t^{\operatorname{deg}_{\theta}(a)}}{a}-1\right) \geq 1 \text {. }
$$

Thus,

$$
\operatorname{ord}\left(\exp _{\psi}\left(\sum_{a \in A_{+}} \frac{\mu(a) a\left(z_{1}\right) \ldots a\left(z_{n}\right) t^{\operatorname{deg}_{\theta}(a)}}{a}\right)-1\right) \geq 1 .
$$

But by Proposition 3.6, we know that the left hand side of (37) is in $A\left[z_{1}, \ldots, z_{n}, t\right]$. Therefore, by the inequality in (41), we get the first part of the theorem. Evaluating (37) at $t=1$ gives the second part of the theorem.

Recall that $\log _{\phi}=\sum_{j \geq 0} \gamma_{j} \tau^{j}$ is the logarithm series corresponding to $\phi$, and $\log _{\psi}(g)=$ $\sum_{j \geq 0} \gamma_{j} \ell_{j}\left(z_{1}\right) \ldots \ell_{j}\left(z_{n}\right) t^{j} \tau^{j}(g)$ for any $g \in \widetilde{\mathbb{T}_{n, t}}$ in the domain of $\log _{\psi}$. We define a positive integer $i(\phi)$ which satisfies

$$
\frac{\operatorname{deg}_{\theta}\left(\phi_{\theta, i(\phi)}\right)-q^{i(\phi)}}{q^{i(\phi)}-1} \geq \frac{\operatorname{deg}_{\theta}\left(\phi_{\theta, s}\right)-q^{s}}{q^{s}-1}
$$

where $s$ runs over all the indices such that $\phi_{\theta, s} \neq 0$. By [12, Cor. 6.9], we have that

$$
\left|\gamma_{j}\right|_{\infty} \leq q^{\frac{q^{j}-1}{q^{i(\phi)}-1}\left(\operatorname{deg}_{\theta}\left(\phi_{\theta, i(\phi)}\right)-q^{i(\phi)}\right)} .
$$

Since $\left\|\ell_{1}\left(z_{k}\right)\right\|=q,\left\|\ell_{j}\left(z_{k}\right)\right\|=q^{1+q+\cdots+q^{j-1}}$ for all $j \geq 2$, and $1 \leq k \leq n$, we have

$$
\left\|\gamma_{j}^{\prime}\right\|=\left\|\gamma_{j} \ell_{j}\left(z_{1}\right) \ldots \ell_{j}\left(z_{n}\right) t^{j}\right\| \leq q^{\frac{q^{j}-1}{q^{i(\phi)}-1}\left(\operatorname{deg}_{\theta}\left(\phi_{i(\phi)}\right)-q^{i(\phi)}+n\left(1+q+\cdots+q^{i(\phi)-1}\right)\right)}
$$

for all $j \geq 1$. Using (42) and properties of non-archimedian norm $\|\cdot\|$, we deduce the following proposition. 
Proposition 4.2. The logarithm function $\log _{\psi}$ converges for all $f \in \widetilde{\mathbb{T}_{n, t}}$ such that

$$
\|f\|<q^{\frac{q^{i(\phi)}-\operatorname{deg}_{\theta}\left(\phi_{\theta, i(\phi)}\right)-n\left(1+q+\cdots+q^{i(\phi)-1}\right)}{q^{i(\phi)}-1}} .
$$

Now, we analyze the value of $L\left(\phi^{\vee}, z_{1}, \ldots, z_{n}, 1\right)$ in the following result.

Corollary 4.3. If $0 \leq n \leq q / r-(1+2 \beta)$, then

$$
\sum_{a \in A_{+}} \frac{\mu(a) a\left(z_{1}\right) \ldots a\left(z_{n}\right) t^{\operatorname{deg}_{\theta}(a)}}{a}=\log _{\psi}(1) .
$$

In particular,

$$
\sum_{a \in A_{+}} \frac{\mu(a) a\left(z_{1}\right) \ldots a\left(z_{n}\right)}{a}=\log _{\varphi}(1)=\frac{\log _{\phi}\left(\omega_{n}\right)}{\omega_{n}} .
$$

Proof. First we show that 1 is within the radius of convergence of $\log _{\psi}$. We again note by (40) that if $0 \leq n \leq q / r-(1+2 \beta)$, then $\beta+n \leq-1+(n+1) / q+(q-1) / r$ and $q>n$. Thus, by the choice of $\beta$ and the inequality $q>n$, we have that

$$
\begin{aligned}
\operatorname{deg}_{\theta}\left(\phi_{\theta, i(\phi)}\right)+n\left(1+q+\cdots+q^{i(\phi)-1}\right) & \leq \frac{q-1}{r}-(n+1)+\frac{n}{q}+\frac{1}{q}+n+n\left(q+\cdots+q^{i(\phi)-1}\right) \\
& <q+n\left(q+\cdots+q^{i(\phi)-1}\right) \\
& \leq q+(q-1)\left(q+\cdots+q^{i(\phi)-1}\right)=q^{i(\phi)} .
\end{aligned}
$$

Therefore, by Proposition 4.2, we see that 1 is within the radius of convergence of $\log _{\psi}$. Finally, applying $\log _{\psi}$ to both sides of (37) finishes the first part. We now prove the last assertion. If we evaluate (43) at $t=1$, then we get the first equality in (44). Now, recall the infinite product expansion of $\omega_{n}$ in (77), and observe that $\tau^{i}\left(\omega_{n}\right)=\ell_{i}\left(z_{1}\right) \ell_{i}\left(z_{2}\right) \cdots \ell_{i}\left(z_{n}\right) \omega_{n}$ for all $i \geq 1$. Thus,

$$
\begin{aligned}
\log _{\phi}\left(\omega_{n}\right) & =\gamma_{0} \omega_{n}+\gamma_{1} \tau\left(\omega_{n}\right)+\gamma_{2} \tau^{2}\left(\omega_{n}\right)+\ldots \\
& =\gamma_{0} \omega_{n}+\gamma_{1} \ell_{1}\left(z_{1}\right) \ell_{1}\left(z_{2}\right) \ldots \ell_{1}\left(z_{n}\right) \omega_{n}+\gamma_{2} \ell_{2}\left(z_{1}\right) \ell_{2}\left(z_{2}\right) \ldots \ell_{2}\left(z_{n}\right) \omega_{n}+\ldots \\
& =\omega_{n} \log _{\varphi}(1)
\end{aligned}
$$

which gives the second equality in (44).

Remark 4.4. Observe that the second equality in Corollary 1.2 follows from the same calculation in (46) replacing 1 by $P_{\phi}\left(z_{1}, \ldots, z_{n}\right)$.

Remark 4.5. One can note that dealing with $\log _{\psi}$ which is not an entire function is one of the obstacles which bounds us from understanding (44) for any $n$ and any Drinfeld $A$ module $\phi$ over $A$. It would be interesting to understand the value of $L\left(\phi^{\vee}, z_{1}, \ldots, z_{n}, 1\right)$ and the polynomial $P_{\phi}\left(z_{1}, \ldots, z_{n}\right)$ in general.

Example 4.6. If we choose $\phi=\tilde{C}$ in Corollary 4.3 which is defined by $\tilde{C}_{\theta}:=\theta+\ell_{1}\left(z_{1}\right) \tau$, then we recover an immediate consequence of [3, Prop. 5.9] which can be stated as

$$
L(\tilde{C}, \mathbb{A})=\sum_{a \in A_{+}} \frac{a\left(z_{1}\right)}{a}=\log _{\tilde{C}}(1)=\frac{\log _{C}\left(\omega_{1}\right)}{\omega_{1}}=-\frac{\tilde{\pi}}{\left(z_{1}-\theta\right) \omega_{1}}
$$


where the last equation follows from the fact that $\omega_{1}=\exp _{C}\left(\tilde{\pi} /\left(\theta-z_{1}\right)\right)$ which was proved by Pellarin in [20, $\S 4]$.

Remark 4.7. Let $\psi$ be a Drinfeld $\tilde{\mathbb{A}}$-module as in Corollary 4.3 and recall that $\log _{\psi}=$ $\sum_{i \geq 0} \gamma_{i} \ell_{i}\left(z_{1}\right) \ldots \ell_{i}\left(z_{n}\right) t^{i} \tau^{i}$ is the logarithm series corresponding to $\psi$. If we compare the coefficients of $t^{i}$ for all $i$ on both sides of (43), we get

$$
\sum_{a \in A_{+, i}} \frac{\mu(a) a\left(z_{1}\right) \ldots a\left(z_{n}\right)}{a}=\gamma_{i} \ell_{i}\left(z_{1}\right) \ldots \ell_{i}\left(z_{n}\right) .
$$

We note that (47) can be seen as the generalization of the formulas obtained by Perkins [21, Thm. 4.16] which relates the coefficients of the logarithm function $\log _{C}$ to Pellarin $L$-Series.

Remark 4.8. We recall the definition of the Carlitz module $C$ from Section 2, and for any $x \in \mathbb{C}_{\infty}$ in the domain of $\log _{C}$, let $\log _{C}(x)=\sum_{i \geq 0} L_{i} \tau^{i}(x)$. For integers $k, i \geq 0$, the power sum $S_{i}(k)$ is defined by

$$
S_{i}(k):=\sum_{a \in A_{+, i}} a^{k} .
$$

Then, Lee proved [18, Thm. 4.1] (see also [23, Cor. 5.6.4(1)]) that

$$
S_{i}\left(q^{k}-1\right)=\sum_{a \in A_{+, i}} a^{q^{k}-1}= \begin{cases}L_{i}\left(\theta^{q^{k}}-\theta\right) \ldots\left(\theta^{q^{k}}-\theta^{q^{i-1}}\right) & \text { if } k \geq i \\ 0 & \text { if } k<i .\end{cases}
$$

We refer the reader to [19] and [23, $\S 5]$ for more details about the sum $S_{i}(k)$.

Now, let $\phi$ be a Drinfeld $A$-module of rank $r$ defined as in (1) such that $0 \leq \beta \leq q /(2 r)-1$, and consider the logarithm series $\log _{\phi}=\sum_{i>0} \gamma_{i} \tau^{i}$ corresponding to $\phi$. If we define the Drinfeld $\tilde{\mathbb{A}}$-module $\psi$ by $\psi_{\theta}=\sum_{i=0}^{r} \phi_{\theta, i} \ell_{i}\left(z_{1}\right) t^{i}$, and evaluate $\log _{\psi}(1)=\sum_{i \geq 0} \gamma_{i} \ell_{i}\left(z_{1}\right) t^{i}$ at $z_{1}=\theta^{q^{k}}$ for some $k \in \mathbb{N}$, then comparing coefficients of $t^{i}$ on both sides of (43) for all $i \geq 0$ implies that

$$
\sum_{a \in A_{+, i}} \mu(a) a^{q^{k}-1}= \begin{cases}\gamma_{i}\left(\theta^{q^{k}}-\theta\right) \ldots\left(\theta^{q^{k}}-\theta^{q^{i-1}}\right) & \text { if } k \geq i \\ 0 & \text { if } k<i\end{cases}
$$

which can be seen as a generalization of (48).

\section{EntiREnEss of THE $L$-SERIES $L\left(\phi^{\vee}, z_{1}, \ldots, z_{n} ; x, y\right)$}

Before we state the main result of this section, we need a definition due to Goss.

Definition. [16, $\S 8.5]$ For each $y \in \mathbb{Z}_{p}$, let $g_{y}(z)$ be a power series in $z$ such that $g_{y}$ converges at all values of $z \in \mathbb{C}_{\infty}$. Let $(x, y) \in \mathbb{S}_{\infty}$. We call the entire power series $f(x, y):=g_{y}(1 / x)$ an entire function on $\mathbb{S}_{\infty}$ if for any bounded subset $H \subset \mathbb{C}_{\infty}$ and $\epsilon>0$, there exists $\delta_{H}>0$ such that if $y_{0}, y_{1} \in \mathbb{Z}_{p}$ and $\left|y_{0}-y_{1}\right|_{p}<\delta_{H}$, then $\left|g_{y_{0}}(z)-g_{y_{1}}(z)\right|_{\infty}<\epsilon$ for all $z \in H$.

This section occupies the proof of the following result.

Theorem 5.1. The infinite series $L\left(\phi^{\vee}, z_{1}, \ldots, z_{n} ; x, y\right)$ can be analytically continued to an entire function on $\mathbb{C}_{\infty}^{n} \times \mathbb{S}_{\infty}$. In particular, the L-series $L\left(\phi^{\vee}, z_{1}, \ldots, z_{n}\right.$, s) converges in $\mathbb{T}_{n}$ for all $s \in \mathbb{Z}$. 
In order to prove Theorem [5.1, we first introduce the following setting in [3, $\S 8]$ which was given by Anglès, Pellarin, and Tavares Ribeiro. We also use Proposition 3.6 to prove Theorem 5.3.

Let $Y_{1}, \ldots, Y_{n}$ be indeterminates. We set

$$
\mathbb{B}_{n}:=\mathbb{C}_{\infty}\left[Y_{1}, \ldots, Y_{n}, \tau\left(Y_{1}\right), \ldots, \tau\left(Y_{n}\right), \ldots\right],
$$

where the action of $\tau$ on $\mathbb{B}_{n}$ is given by $\tau \cdot \tau^{i}\left(Y_{j}\right)=\tau^{i+1}\left(Y_{j}\right)$. Let $W$ be another indeterminate. We define $\mathbb{D}_{n}$ to be the ring of elements of the form $\sum_{i \geq 0} b_{i} \tau^{i}(W)$, where $b_{i} \in \mathbb{B}_{n}$. For elements $F=\sum f_{i} \tau^{i}(W)$ and $G=\sum g_{i} \tau^{i}(W)$ in $\mathbb{D}_{n}$, the multiplication $F \cdot G$ is given by $\sum_{k \geq 0} \sum_{i+j=k} f_{i} \tau^{i}\left(g_{j}\right) \tau^{k}(W)$, and the action of $\tau$ on $\tau^{i}(W)$ is equal to $\tau^{i+1}(W)$. Using the Drinfeld $A$-module $\phi$ in (1), define

$$
\mathscr{E}_{\phi}\left(Y_{1}, \ldots, Y_{n}, W\right):=\sum_{a \in A_{+}} \frac{\mu(a) C_{a}\left(Y_{1}\right) \ldots C_{a}\left(Y_{n}\right)}{a} \tau^{\operatorname{deg}_{\theta}(a)}(W) .
$$

The multiplication rule of the elements in $\mathbb{D}_{n}$ by any element $\gamma \in K, z_{j}$ for all $1 \leq j \leq n$, and $t$ is given as follows:

$$
\begin{aligned}
\gamma \cdot f & =\gamma f \text { for any } f \in \mathbb{D}_{n} \\
z_{j} \cdot \tau^{m}\left(Y_{i}\right) & =\tau^{m}\left(Y_{i}\right) \text { if } i \neq j \\
z_{j} \cdot \tau^{m}\left(Y_{j}\right) & =\tau^{m}\left(C_{\theta}\left(Y_{j}\right)\right) \text { for all } 1 \leq j \leq n \\
t \cdot \tau^{m}\left(Y_{j}\right) & =\tau^{m}\left(Y_{j}\right) \text { for all } 1 \leq j \leq n \\
z_{j} \cdot \tau^{m}(W) & =\tau^{m}(W) \text { for all } 1 \leq j \leq n \\
t \cdot \tau^{m}(W) & =\tau^{m+1}(W) .
\end{aligned}
$$

Moreover, for any $f \in \mathbb{D}_{n}$ and $g \in K\left[z_{1}, \ldots, z_{n}, t\right]$ given by

$$
g=\sum_{j_{1}, \ldots, j_{n}, j_{n+1} \geq 0} g_{j_{1} \ldots j_{n+1}} z_{1}^{j_{1}} \ldots z_{n}^{j_{n}} t^{j_{n+1}}
$$

we define the $K\left[z_{1}, \ldots, z_{n}, t\right]$-action on $\mathbb{D}_{n}$ by

$$
g \cdot f=\sum_{j_{1}, \ldots, j_{n}, j_{n+1} \geq 0}\left(z_{1}^{j_{1}} \cdot f\right) \ldots\left(z_{n}^{j_{n}} \cdot f\right)\left(t^{j_{n+1}} \cdot f\right) .
$$

Thus, the ring $\mathbb{D}_{n}$ is now a $K\left[z_{1}, \ldots, z_{n}, t\right]$-algebra. Using (49), we conclude that for any $a=a(\theta) \in A$ and any $m \geq 1$, we have

$$
\left(a\left(z_{1}\right) \ldots a\left(z_{n}\right)\right) \cdot \tau^{m}\left(Y_{1} \ldots Y_{n}\right)=\tau^{m}\left(C_{a}\left(Y_{1}\right) \ldots C_{a}\left(Y_{n}\right)\right) .
$$

The action of $K\left[z_{1}, \ldots, z_{n}\right]$ to $\mathbb{D}_{n}$ extends to an action of $K\left[z_{1}, \ldots, z_{n}\right][[t]]$ in a following way: If $G=\sum G_{i} t^{i} \in K\left[z_{1}, \ldots, z_{n}\right][[t]]$, we set

$$
G \cdot\left(Y_{1} \ldots Y_{n} W\right):=\sum G_{i} \cdot\left(Y_{1} \ldots Y_{n} \tau^{i}(W)\right) .
$$

We have the following useful lemma for the action defined above.

Lemma 5.2 (Anglès, Pellarin, Tavares Ribeiro [4, Lem. 3.11(2)]). The map $g: K\left[z_{1}, \ldots, z_{n}, t\right] \rightarrow$ $\mathbb{D}_{n}$ defined by

$$
g(f)=f \cdot\left(Y_{1} \ldots Y_{n}\right)
$$

is injective for all $f \in K\left[z_{1}, \ldots, z_{n}, t\right]$. 
By Theorem 3.5, the Taelman $L$-value $L(\psi, \tilde{\mathbb{A}})$ corresponding to Drinfeld $\tilde{\mathbb{A}}$-module $\psi$ defined in (31) is given by

$$
L(\psi, \tilde{\mathbb{A}})=\sum_{a \in A_{+}} \frac{\mu(a) a\left(z_{1}\right) \ldots a\left(z_{n}\right) t^{\operatorname{deg}_{\theta}(a)}}{a},
$$

and by (49) and (50), we get $L(\psi, \tilde{\mathbb{A}}) \cdot\left(Y_{1} \ldots Y_{n} W\right)=\mathscr{E}_{\phi}\left(Y_{1}, \ldots, Y_{n}, W\right)$. By the action defined in (49), we notice that for any $1 \leq j \leq n$,

$$
\begin{aligned}
t^{i} \ell_{i}\left(z_{j}\right) \cdot Y_{j} W & =t^{i}\left(\left(z_{j}-\theta^{q^{i-1}}\right) \ldots\left(z_{j}-\theta^{q}\right)\left(z_{j}-\theta\right)\right) \cdot Y_{j} W \\
& =t^{i}\left(z_{j}-\theta^{q^{i-1}}\right) \ldots\left(z_{j}-\theta^{q^{2}}\right)\left(z_{j}-\theta^{q}\right) \cdot\left(C_{\theta}\left(Y_{j}\right)-\theta Y_{j}\right) W \\
& =t^{i}\left(z_{j}-\theta^{q^{i-1}}\right) \ldots\left(z_{j}-\theta^{q^{2}}\right)\left(z_{j}-\theta^{q}\right) \cdot \tau\left(Y_{j}\right) W \\
& =t^{i}\left(z_{j}-\theta^{q^{i-1}}\right) \ldots\left(z_{j}-\theta^{q^{2}}\right) \cdot\left(\tau\left(C_{\theta}\left(Y_{j}\right)\right)-\theta^{q} Y_{j}\right) W \\
& =t^{i}\left(z_{j}-\theta^{q^{i-1}}\right) \ldots\left(z_{j}-\theta^{q^{2}}\right) \cdot \tau^{2}\left(Y_{j}\right) W \\
& \vdots \\
& =t^{i} \tau^{i}\left(Y_{j}\right) W \\
& =\tau^{i}\left(Y_{j} W\right)
\end{aligned}
$$

and therefore (151) implies that

$$
\begin{aligned}
t^{i} \ell_{i}\left(z_{j}\right) \cdot\left(Y_{1} Y_{2} \ldots Y_{j-1} Y_{j} Y_{j+1} \ldots Y_{n} W\right) & =t^{i} \ell_{i}\left(z_{j}\right) \cdot\left(Y_{j} Y_{1} \ldots Y_{j-1} Y_{j+1} \ldots Y_{n} W\right) \\
& =t^{i} \cdot \tau^{i}\left(Y_{j}\right) Y_{1} \ldots Y_{j-1} Y_{j+1} \ldots Y_{n} W \\
& =\tau^{i}\left(Y_{j} W\right) Y_{1} \ldots Y_{j-1} Y_{j+1} \ldots Y_{n} .
\end{aligned}
$$

Combining (51) and (52) gives that

$$
t^{i} \ell_{i}\left(z_{1}\right) \ldots \ell_{i}\left(z_{n}\right) \cdot\left(Y_{1} Y_{2} \ldots Y_{j-1} Y_{j} Y_{j+1} \ldots Y_{n}\right)=\tau^{i}\left(Y_{1} \ldots Y_{n} W\right)
$$

Since $\exp _{\psi}=\sum_{i} \alpha_{i} t^{i} \ell_{i}\left(z_{1}\right) \ldots \ell_{i}\left(z_{n}\right) \tau^{i}$, (150) and (53) imply that

$$
\exp _{\phi}\left(\mathscr{E}_{\phi}\left(Y_{1}, \ldots, Y_{n}, W\right)\right)=\exp _{\psi}(L(\psi, \tilde{\mathbb{A}})) \cdot\left(Y_{1} \ldots Y_{n} W\right)
$$

But by Proposition [3.6, $\exp _{\psi}(L(\psi, \tilde{\mathbb{A}})) \in A\left[z_{1}, \ldots, z_{n}, t\right]$. Therefore, we have that

$$
\exp _{\phi}\left(\mathscr{E}_{\phi}\left(Y_{1}, \ldots, Y_{n}, W\right)\right) \in A\left[Y_{1}, \ldots, Y_{n}, W\right] .
$$

Now for any $m \in \mathbb{N}$ and indeterminates $X_{1}, X_{2}, \ldots, X_{n}, w$, choose a suitable $\mathbb{C}_{\infty}$-algebra homomorphism sending $\tau^{m}\left(Y_{j}\right)$ to $X_{j}^{q^{m}}$ for all $1 \leq j \leq n$ and $\tau^{m}(W)$ to $w^{q^{m}}$. Rewriting (54) with this homomorphism, we get the following theorem.

Theorem 5.3. Let $\phi$ be a Drinfeld $A$-module as in (1), and let $X_{1}, \ldots, X_{n}, w$ be indeterminates. The series

$$
\exp _{\phi}\left(\sum_{a \in A_{+}} \frac{\mu(a) C_{a}\left(X_{1}\right) \ldots C_{a}\left(X_{n}\right)}{a} w^{q^{\operatorname{deg}_{\theta}(a)}}\right) \in K\left[X_{1}, \ldots, X_{n}\right][[w]]
$$

is actually in $A\left[X_{1}, \ldots, X_{n}, w\right]$. 
We define

$$
S_{k}^{\phi}=\sum_{a \in A_{+, k}} \frac{\mu(a) C_{a}\left(X_{1}\right) \ldots C_{a}\left(X_{n}\right)}{a}
$$

and

$$
\jmath^{\phi}=\sum_{a \in A_{+}} \frac{\mu(a) C_{a}\left(X_{1}\right) \ldots C_{a}\left(X_{n}\right)}{a} w^{q^{\operatorname{deg}(a)}}=\sum_{k \geq 0} S_{k}^{\phi} w^{q^{k}} .
$$

For $\exp _{\phi}=\sum_{j \geq 0} \alpha_{j} \tau^{j}$, we set $Z_{k}^{\phi}=\sum_{i=0}^{k}\left(S_{k-i}^{\phi}\right)^{q^{i}} \alpha_{i}$, and therefore

$$
\exp _{\phi}\left(\jmath^{\phi}\right)=\sum_{k \geq 0} Z_{k}^{\phi} w^{q^{k}}
$$

By Theorem 5.3 and (55), we have $Z_{k}^{\phi} \in A\left[X_{1}, \ldots, X_{n}\right]$ for all $k \geq 0$, and for $k$ arbitrarily large, $Z_{k}^{\phi}=0$.

We recall the definition of the Carlitz module $C$ from Section 2 and that $\operatorname{Ker}\left(\exp _{C}\right)=$ $\tilde{\pi} A$ where $\tilde{\pi} \in \mathbb{C}_{\infty}^{\times}$. Let $\tilde{X}$ be the set of indeterminates $X_{1}, \ldots, X_{n}$. For indeterminates $X_{1}, \ldots X_{n}$, we denote the polynomial ring $\mathbb{C}_{\infty}\left[X_{1}, \ldots, X_{n}\right]$ by $\mathbb{C}_{\infty}[\tilde{X}]$. Following the work of Anglès, Pellarin, and Tavares Ribeiro in $[4, \S 3]$, for any $G \in \mathbb{C}_{\infty}[\tilde{X}]$, we define a function $\|\cdot\|_{\tilde{X}}: \mathbb{C}_{\infty}[\tilde{X}] \rightarrow \mathbb{R}$ by

$$
\|G\|_{\tilde{X}}=\sup \left\{\left|G\left(x_{1}, \ldots, x_{n}\right)\right|_{\infty} \mid x_{i} \in \exp _{C}\left(K_{\infty} \tilde{\pi}\right) \quad \text { for } 1 \leq i \leq n\right\}
$$

so that $\|\cdot\|_{\tilde{X}}$ defines a well-defined and ultrametric norm on $\mathbb{C}_{\infty}[\tilde{X}]$ (see [4, $\left.\S 3\right]$ ).

Proposition 5.4. Let $\phi$ be a Drinfeld $A$-module of rank $r$ as in (1) and $\beta=\max \left\{\operatorname{deg}_{\theta}\left(\phi_{\theta, i}\right) \mid\right.$ $1 \leq i \leq r\}$. Then $Z_{k}^{\phi}=0$ when $k>r(n+\beta) /(q-1)$.

Proof. We apply the similar methods in the proof of [므, Thm. 6.2]. We know by [4, Lem. 3.1(1)] that $\left\|X_{1} \ldots X_{n}\right\|_{\tilde{X}}=q^{n / q-1}$. Note that by [4, §3.1], for all $a \in A$ and $G \in \mathbb{C}_{\infty}[\tilde{X}]$, the action $a \cdot G=G\left(C_{a}\left(X_{1}\right), \ldots, C_{a}\left(X_{n}\right)\right)$ is isometric with respect to the norm $\|\cdot\|_{\tilde{X}}$. Thus, we have

$$
\left\|C_{a}\left(X_{1}\right) C_{a}\left(X_{2}\right) \ldots C_{a}\left(X_{n}\right)\right\|_{\tilde{X}}=\left\|X_{1} \ldots X_{n}\right\|_{\tilde{X}}=q^{n / q-1}
$$

By [11, Eq. 28], we get

$$
\left|\alpha_{k}\right|_{\infty} \leq q^{q^{k}(\beta /(q-1)-k / r)}
$$

The definition of $S_{k}^{\phi}$, Lemma $2.2(\mathrm{~b})$ and (156) imply

$$
\left\|S_{k}^{\phi}\right\|_{\tilde{X}} \leq \max _{a \in A_{+, k}}\left\{\left\|\frac{\mu(a)}{a}\right\|\left\|X_{1} \ldots X_{n}\right\|_{\tilde{X}}\right\} \leq q^{-k / r} q^{n / q-1} .
$$

Therefore, (57) and (58) imply that

$$
\left\|Z_{k}^{\phi}\right\|_{\tilde{X}} \leq \max _{0 \leq i \leq k}\left\{\left\|S_{k-i}^{\phi}\right\|_{\tilde{X}}^{q^{i}}\left|\alpha_{i}\right|_{\infty}\right\} \leq\left(q^{n / q-1}\right)^{q^{k}} q^{q^{k}(\beta /(q-1)-k / r)}=q^{q^{k}\left(\frac{n+\beta}{q-1}-\frac{k}{r}\right)} .
$$

Finally, when $k>r(n+\beta) /(q-1)$, we have $\left\|Z_{k}^{\phi}\right\|_{\tilde{X}}<1$ which implies that $Z_{k}^{\phi}=0$ by [4, Lem. 4.3].

Lemma 5.5. For $k>r(n+\beta) /(q-1)$, the series $H_{k, n-1}:=\sum_{a \in A_{+, k}} \mu(a) a\left(z_{1}\right) \ldots a\left(z_{n-1}\right)$ vanishes. In particular, $L\left(\phi^{\vee}, z_{1}, \ldots, z_{n-1}, 0\right) \in A\left[z_{1}, \ldots, z_{n-1}\right]$. 
Proof. The proof is using the ideas of the proof of [4, Lem. 4.13]. If we see $S_{k}^{\phi}$ as a polynomial of $X_{n}$ without any constant term, and note that $C_{a}\left(X_{n}\right) \equiv a X_{n}\left(\bmod X_{n}^{q}\right)$, we have

$$
\begin{aligned}
Z_{k}^{\phi} \equiv S_{k}^{\phi} & \equiv \sum_{a \in A_{+, k}} \frac{\mu(a) C_{a}\left(X_{1}\right) \ldots C_{a}\left(X_{n}\right)}{a} \quad\left(\bmod X_{n}^{q}\right) \\
& \equiv \sum_{a \in A_{+, k}} \frac{\mu(a) C_{a}\left(X_{1}\right) \ldots C_{a}\left(X_{n-1}\right) a X_{n}}{a} \quad\left(\bmod X_{n}^{q}\right) \\
& \equiv X_{n} \sum_{a \in A_{+, k}} \mu(a) C_{a}\left(X_{1}\right) \ldots C_{a}\left(X_{n-1}\right) \quad\left(\bmod X_{n}^{q}\right)
\end{aligned}
$$

For $k>r(n+\beta) /(q-1)$, we know by Proposition 5.4 that $Z_{k}^{\phi}=0$ which gives us that

$$
\sum_{a \in A_{+, k}} \mu(a) C_{a}\left(X_{1}\right) \ldots C_{a}\left(X_{n-1}\right)=0 .
$$

Moreover by (150), we have that

$$
\sum_{a \in A_{+, k}} \mu(a) C_{a}\left(X_{1}\right) \ldots C_{a}\left(X_{n-1}\right)=\sum_{a \in A_{+, k}} \mu(a) a\left(z_{1}\right) \ldots a\left(z_{n-1}\right) \cdot\left(X_{1} \ldots X_{n-1}\right) .
$$

By Lemma 5.2, (60) and (61), we conclude that

$$
\sum_{a \in A_{+, k}} \mu(a) a\left(z_{1}\right) \ldots a\left(z_{n-1}\right)=0
$$

for $k>r(n+\beta) /(q-1)$. On the other hand, we have

$$
L\left(\phi^{\vee}, z_{1}, \ldots, z_{n-1}, 0\right)=\sum_{k \geq 0} \sum_{a \in A_{+, k}} \mu(a) a\left(z_{1}\right) \ldots a\left(z_{n-1}\right) .
$$

Therefore, the second part of the proposition follows from the first part.

Remark 5.6. We note that Lemma 5.5] can be seen as a corollary of Simon's Lemma [2, Lem. 4] when $\phi=C$.

Lemma 5.7. For $d \geq 3 r+r(n+1+\beta) /(q-1)$ and $n>0$, we have

$$
\left\|\mathscr{L}_{d, n}(x, y)\right\| \leq|x|_{\infty}^{-d} q^{d\left(1-\frac{1}{r}\right)} q^{-q^{\left[\frac{d}{r}-\frac{n+1+\beta}{q-1}\right]-2}},
$$

where [·] is the integer part function.

Proof. We adapt the ideas in [2, Lem. 7]. Recall from Section 1 that $p=q^{l}$. For any $w \in \mathbb{Z}_{p}$ such that $w=\sum w_{i} q^{i}$ where $0 \leq w_{i} \leq q-1$, set $\ell_{q}(w):=\sum w_{i}$. Let $y \in \mathbb{Z}_{p}$, and $\sum_{j \geq 0} b_{j} p^{j}$ be the $p$-adic expansion of $y$ such that $0 \leq b_{j} \leq p-1$ for all $j$, and for $m \geq 1$, set $y_{m}:=\sum_{i=0}^{l m-1} b_{k} p^{k}$ such that $\sum_{j=0}^{m} v_{j} q^{j}$ be the $q$-adic expansion of $y_{m}$ where $0 \leq v_{j} \leq q-1$. We have that

$$
\mathscr{L}_{d, n}\left(x, y_{m}\right)=\frac{1}{x^{d} \theta^{d y_{m}}} H_{d, n+\ell_{q}\left(y_{m}\right)}\left(z_{1}, \ldots, z_{n}, \theta, \ldots, \theta, \ldots, \theta^{q^{m}}, \ldots, \theta^{q^{m}}\right),
$$

where $\theta^{q^{i}}$ appears $v_{i}$ many times. By the bound on coefficients $v_{j}$, it is easy to see that

$$
\ell_{q}\left(y_{m}\right)=\sum_{j=0}^{m} v_{j} \leq(m+1)(q-1)
$$


By Lemma 5.5, if $d>r(n+1+(m+1)(q-1)+\beta) /(q-1)=r(m+1)+r(n+\beta+1) /(q-1)$, then $H_{d, n+\ell_{q}\left(y_{m}\right)}\left(x, y_{m}\right)=0$. Notice that by the definition of $y_{m}$ and (36) $)$, we have that

$$
\left(\begin{array}{c}
y \\
j
\end{array}\right)=\left(\begin{array}{c}
y_{m} \\
j
\end{array}\right)
$$

for $j=0,1, \ldots, q^{m}-1$. Therefore, for any $a \in A_{+, d}$,

$$
\left|\sum_{j \geq 0}\left(\left(\begin{array}{c}
y \\
j
\end{array}\right)-\left(\begin{array}{c}
y_{m} \\
j
\end{array}\right)\right)(\langle a\rangle-1)^{j}\right|_{\infty} \leq q^{-q^{m}}
$$

Thus by Lemma 2.2(b) and (62), we have that

$$
\begin{aligned}
\left\|\mathscr{L}_{d, n}(x, y)-\mathscr{L}_{d, n}\left(x, y_{m}\right)\right\| & =\left\|x^{-d} \sum_{a \in A_{+, d}} \mu(a) a\left(z_{1}\right) \ldots a\left(z_{n}\right) \sum_{i \geq 0}\left(\left(\begin{array}{c}
y \\
i
\end{array}\right)-\left(\begin{array}{c}
y_{m} \\
i
\end{array}\right)\right)(\langle a\rangle-1)^{i}\right\| \\
& \leq|x|_{\infty}^{-d} q^{d\left(1-\frac{1}{r}\right)} q^{-q^{m}} .
\end{aligned}
$$

If we choose $m+2=[d / r-(n+\beta+1) /(q-1)] \geq 3$, then

$$
\mathscr{L}_{d, n}\left(x, y_{m}\right)=\frac{1}{\theta^{d y_{m}}} H_{d, n+\ell_{q}\left(y_{m}\right)}\left(z_{1}, \ldots, z_{n}, \theta, \ldots, \theta, \ldots, \theta^{q^{m}}, \ldots, \theta^{q^{m}}\right)=0 .
$$

Therefore, the result follows from (63) and our choice for $m$ above.

Proof of Theorem 5.1. Let $(x, y) \in \mathbb{S}_{\infty}$. For any $\xi_{i} \in \mathbb{C}_{\infty}$ and arbitrarily large $d$, we have by Lemma 5.7 that

$$
\left\|\sum_{a \in A_{+, d}} \mu(a) a\left(\xi_{1}\right) \ldots a\left(\xi_{n}\right)\langle a\rangle^{y}\right\| \leq\left|\xi_{1} \ldots \xi_{n}\right|_{\infty}^{d} q^{d\left(1-\frac{1}{r}\right)} q^{-q^{\left[\frac{d}{r}-\frac{n+1+\beta}{q-1}\right]-2}} .
$$

Thus, we see that as $d$ goes to $\infty$, the right hand side of (64) approaches to 0 . On the other hand, we have that

$$
L\left(\phi^{\vee}, z_{1}, \ldots, z_{n} ; x, y\right)=\sum_{d \geq 0} x^{-d} \sum_{a \in A_{+, d}} \mu(a) a\left(z_{1}\right) \ldots a\left(z_{n}\right)\langle a\rangle^{y} .
$$

Therefore, by [16, Thm. 8.5.7], the series $L\left(\phi^{\vee}, z_{1}, \ldots, z_{n} ; x, y\right)$ is entire on $\mathbb{C}_{\infty}^{n} \times \mathbb{S}_{\infty}$ which proves the theorem.

Remark 5.8. A natural direction at this point would be understanding the zeros of the $L$-series $L\left(\phi^{\vee}, z_{1}, \ldots, z_{n} ; x, y\right)$. When $\phi$ is the Carlitz module $C$, we refer the reader to results explained in [16]. For a Drinfeld $A$-module of higher rank, studying zeroes of $L\left(\phi^{\vee}, z_{1}, \ldots, z_{n} ; x, y\right)$ seems challenging because of the function $\mu: A_{+} \rightarrow A$ whose nature has not been discovered deeply yet. We hope to turn back this problem in future.

\section{REFERENCES}

[1] G. W. Anderson, Log-algebraicity of twisted A-harmonic series and special values of L-series in characteristic $p$, J. Number Theory 60 (1996), no. 1, 165-209.

[2] B. Anglès and F. Pellarin, Functional identites for L-series values in positive characteristic, J. Number Theory 142 (2014), 223-251.

[3] B. Anglès, F. Pellarin, and F. Tavares Ribeiro, Arithmetic of positive characteristic L-series values in Tate algebras. With an appendix by F. Demeslay, Compos. Math. 152 (2016), no. 1, 1-61. 
[4] B. Anglès, F. Pellarin, and F. Tavares Ribeiro, Anderson-Stark units for $\mathbb{F}_{q}[\theta]$, Trans. Amer. Math. Soc., 370 (2018), no. 3, 1603-1627.

[5] B. Anglès and L. Taelman, Arithmetic of characteristic $p$ special L-values. With an appendix by V. Bosser, Proc. Lond. Math. Soc. (3) 110 (2015), no. 4, 1000-1032.

[6] B. Anglès and F. Tavares Ribeiro, Arithmetic of function field units, Math. Ann. 367 (2017), 501-579.

[7] W. D. Brownawell, Linear independence and divided derivative of a Drinfeld module. I. in: Number Theory in Progress, Vol. 1 (Zakopane-Kościelisko, 1997), de Gruyter, Berlin, 1999, pp.47-61.

[8] C.-Y. Chang, A. El-Guindy, and M. A. Papanikolas Log-algebraic identities on Drinfeld modules and special L-values, J. London Math. Soc. (2) 97 (2018), 125-144.

[9] F. Demeslay, A class formula for L-series in positive characteristic, arXiv:1412.3704, 2014.

[10] D. S. Dummit and R. M. Foote Abstract Algebra, John Wiley \& Sons, Inc., 2004.

[11] A. El-Guindy and M. A. Papanikolas, Explicit formulas for Drinfeld modules and their periods, J. Number Theory 133 (2013), no. 6, 1864-1886.

[12] A. El-Guindy and M. A. Papanikolas, Identities for Anderson generating functions for Drinfeld modules, Monatsh. Math. 173 (2014), no. 3-4, 471-493.

[13] N. J. Fine, Binomial coefficients modulo a prime, Amer. Math. Monthly 54 (1947), no. 10, 589-592.

[14] E.-U. Gekeler, On finite Drinfeld modules, J. Algebra 141 (1991), no. 2, 167-182.

[15] O. Gezmiş and M. A. Papanikolas, The de Rham isomorphism for Drinfeld modules over Tate algebras, arXiv:1805.05386, 2018.

[16] D. Goss, Basic Structures of Function Field Arithmetic, Springer-Verlag, Berlin, 1996.

[17] D. Goss, On the L-series of F. Pellarin, J. Number Theory 133 (2012), 955-962.

[18] H. L. Lee, Power sums of polynomials in a Galois field, Duke Math. J. 10 (1943), no. 2, 277-292.

[19] M. A. Papanikolas, Log-algebraicity on tensor powers of the Carlitz module and special values of Goss $L$-functions, In preparation.

[20] F. Pellarin, Values of certain L-series in positive characteristic, Ann. of Math. (2) 176 (2012), no. 3, 2055-2093.

[21] R. Perkins, On Pellarin L-series, Proc. Amer. Math. Soc. 142 (2014), 3355-3368.

[22] L. Taelman, Special L-values of Drinfeld modules, Ann. Math. 75 (2012), 369-391.

[23] D. S. Thakur, Function Field Arithmetic, World Scientific Publishing, River Edge, NJ, 2004.

Department of Mathematics, Texas A\&M University, College Station, TX 77843, U.S.A. E-mail address: oguz@math.tamu.edu 